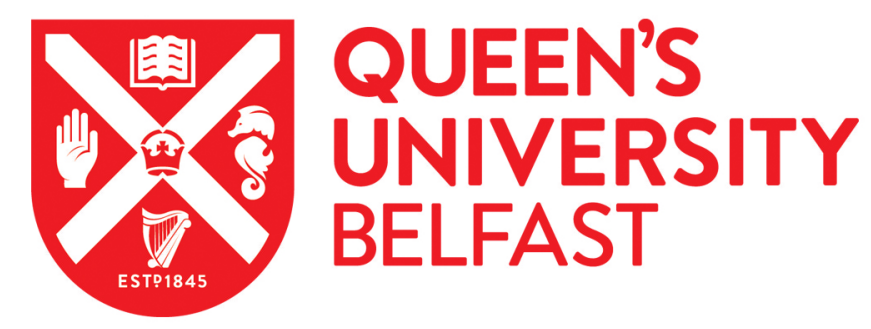

\title{
Stiffness Analysis and Experiment of a Novel 5-DoF Parallel Kinematic Machine Considering Gravitational Effects
}

\author{
Lian, B., Sun, T., Song, Y., Jin, Y., \& Price, M. (2015). Stiffness Analysis and Experiment of a Novel 5-DoF \\ Parallel Kinematic Machine Considering Gravitational Effects. International Journal of Machine Tools and \\ Manufacture, 95, 82-96. https://doi.org/10.1016/j.ijmachtools.2015.04.012
}

Published in:

International Journal of Machine Tools and Manufacture

Document Version:

Early version, also known as pre-print

Queen's University Belfast - Research Portal:

Link to publication record in Queen's University Belfast Research Portal

Publisher rights

Copyright 2015 The Author(s)

\section{General rights}

Copyright for the publications made accessible via the Queen's University Belfast Research Portal is retained by the author(s) and / or other copyright owners and it is a condition of accessing these publications that users recognise and abide by the legal requirements associated with these rights.

Take down policy

The Research Portal is Queen's institutional repository that provides access to Queen's research output. Every effort has been made to ensure that content in the Research Portal does not infringe any person's rights, or applicable UK laws. If you discover content in the Research Portal that you believe breaches copyright or violates any law, please contact openaccess@qub.ac.uk. 


\title{
Stiffness Analysis and Experiment of a Novel 5-DoF Parallel Kinematic Machine Considering Gravitational Effects
}

\author{
Binbin Lian ${ }^{\mathrm{a}}$, Tao Sun ${ }^{\mathrm{a}} *$, Yimin Song ${ }^{\mathrm{a}}$, Yan Jin ${ }^{\mathrm{b}}$, Mark Price ${ }^{\mathrm{b}}$ \\ ${ }^{a}$ Key Laboratory of Mechanism Theory and Equipment Design of Ministry of Education, \\ Tianjin University, Tianjin 300072, China \\ ${ }^{\mathrm{b}}$ School of Mechanical and Aerospace Engineering, Queen's University Belfast, BT9 5AH, UK
}

\begin{abstract}
s
In order to carry out high-precision machining of aerospace structural components with large size, thin wall and complex surface, this paper proposes a novel parallel kinematic machine (PKM) and formulates its semi-analytical theoretical stiffness model considering gravitational effects that is verified by stiffness experiments. From the viewpoint of topology structure, the novel PKM consists of two substructures in terms of the redundant and overconstrained parallel mechanisms that are connected by two interlinked revolute joints. The theoretical stiffness model of the novel PKM is established based upon the virtual work principle and deformation superposition principle after mapping the stiffness models of substructures from joint space to operated space by Jacobian matrices and considering the deformation contributions of interlinked revolute joints to two substructures. Meanwhile, the component gravities are treated as external payloads exerting on the end reference point of the novel PKM resorting to static equivalence principle. This approach is proved by comparing the theoretical stiffness values with experimental stiffness values in the same configurations, which also indicates equivalent gravity can be employed to describe the actual distributed gravities in an acceptable accuracy manner. Finally, on the basis of the verified theoretical stiffness model, the stiffness distributions of the novel PKM are illustrated and the contributions of component gravities to the stiffness of the novel PKM are discussed.
\end{abstract}

Keywords: parallel kinematic machine (PKM), stiffness model, gravitational effect, stiffness experiment

\section{Introduction}

In the high-precision machining of aerospace structural components with large size, thin wall and complex surface, a new type of five degree-of-freedom (DoF) numerical control machine, referred to as parallel kinematic machine (PKM), has been paid more and more attention from both academia and industry [1-4]. As an important complement of traditional 5-DoF numerical control machines with serial topology structures, the parallel kinematic machines (PKMs) with closed topology structures have several advantages in terms of high stiffness, good accuracy/dynamic performance, large load-weight ratio and easy integration with long guideways [5-7]. Those have been proved by successful applications of famous Tricept [8-10] and Sprint Z3 head [11-12] PKMs, not only in the aerospace machining but also in fields like automobile and aviation assembling, wielding, drilling

The existing 5-DoF PKMs can be classified into two categories. The first category is composed of a 3-DoF position parallel mechanism (3-UPSS\&UP [13], 3-UPS\&UP [14], 2-UPR\&SR [15] parallel mechanism for example) plus a 2-DoF rotating head

\footnotetext{
*Corresponding author. Tel: +86(0)22 87402015;

E-mail address: stao@tju.edu.cn (T. Sun).
} 
attached to the moving platform, which has been exampled successfully by Tricept PKM. Here, U, P, S and R denote the universal, prismatic, spherical and rotational joints, respectively, $\underline{\mathrm{P}}$ denotes the actuated prismatic joint driven by a servomotor. The second category is the integration of a 3-DoF pose parallel mechanism (3-PRS[16], 3-RㅛS[17] parallel mechanism for example) and two long guideways, which has been exampled successfully by Sprint Z3 Head PKM. The two categories are especially designed for their applicability to high-precision machining of large-scale, thin-wall and complex-surface structural components. However, the 2-DoF rotating head in the first category will be designed towards large size and heavy weight in order to match the stiffness of the 3-DoF position parallel mechanism and then avoid the bucket effect, which will make a great impact on the dynamic performance of these 5-DoF PKMs. Herein, bucket effect means that the weakest part will determine the performance of the whole system. While for the second category, the two long guideways will influence accuracy and cooperation performance of the 5-DoF PKM. Moreover, the parasitic motion in the 3-DoF pose parallel mechanism [18] may lead to the increment of the control cost. Driven by these problems, the authors of this paper proposed a novel 5-DoF PKM (hereafter referred to as 'T5 PKM') [19], whose servomotors are all arranged adjacent to the fixed base to reduce the weight of the 2-DoF rotating head and avoid integration with two long guideways.

As an important factor evaluating the effects of external force and torque on the displacements of the end-effector, stiffness or compliance is one of the vital performances that should be considered for the analysis of above-mentioned 5-DoF PKMs [20-21]. In addition, when applying for high-precision machining of large-scale, thin-wall and complex-surface aerospace structural components, the stiffness or compliance performance will have significant effect on the accuracy performance of these 5-DoF PKMs [22]. It's worth pointing out that the stiffness or compliance modeling is a research hotspot and an essential foundation for the stiffness or compliance analysis, design and estimation in the preliminary and final design stages of 5-DoF PKMs. Literature reviews [23-38] show that there are three main methods for the formulation of stiffness or compliance model: numerical approach based on finite element analysis (FEA) software, analytical approaches based on structural matrix, and semi-analytical approach based on virtual work principle.

The numerical approach based on FEA software is the most accurate and reliable approach to formulate the stiffness or compliance model, in which the components with irregular shape can be modeled precisely and actually [23-24]. However, the stiffness mapping between joint space and operated space cannot be demonstrated explicitly. In addition, the FEA models have to be re-meshed and re-computed when configuration of end-effector changes, which is inevitable to increase the computational costs. Therefore, the numerical approach based on FEA software is not suitable for parameterized stiffness model. It is usually utilized to the stiffness estimation in the preliminary design stage and the stiffness verification in the final design stage.

The analytical approach based on structural matrix uses the kineto-elasto dynamic (KED) method for reference to formulate the stiffness matrix of the parallel mechanism. By combining stiffness matrices of standard elements such as beam, shell and solid elements [23], the stiffness matrix can be obtained by a general step or flow no matter how complicated the parallel mechanism are. For instance, Deblaise [25] and Wu [26] successfully establish the stiffness models of Delta mechanism and a 5-DoF redundant mechanism by means of structural matrix, respectively. However, Pashkevich [24] points out that the analytical approach based on structural matrix is more complicated in computing multi-dimensional matrix and not suitable for stiffness design of parallel mechanisms. In addition, the stiffness matrices of standard elements are not accurate to characterize those of the actual components with irregular shape.

The semi-analytical approach based on virtual work principle is to formulate the force and deformation mapping between joint space and operated space in the light of virtual work equation, and then obtain the stiffness model of parallel mechanisms by applying Jacobian matrix and Hooke's Law. The stiffness values of some components are obtained by means of FEA software [20, 27]. This approach can be traced back to the work of Salisbury in 1980 [28] that map the stiffness of the joint space of a sprinkler mechanism to 
that of the operated space by means of Jacobian matrix. In 1990, Gosslin [29] formulate the stiffness models of planar 3-DoF and spatial 6-DoF parallel mechanisms merely considering the actuator compliances. By taking into account several deformations of components caused by payloads, a more accurate stiffness model of a 6-DoF Stewart platform is obtained by Huang and Kao [30]. Noticing that the Jacobian matrix of lower mobility parallel mechanisms cannot map the stiffness along/about constrained directions, Ceccarelli [31], Zhang [9] and Li [32-33] utilize the virtual spring to describe the limb deformations caused by the constraint forces, and then carried out the stiffness modelling of CaPaMan, Tripod-based, 3-PUU and 3-PRC parallel mechanisms. By considering theoretically accessible and inaccessible instantaneous motions, the generalized Jacobian matrix of lower mobility parallel mechanisms is obtained by Huang [34]. Based upon overall and generalized Jacobian matrices, the stiffness modeling of several lower mobility parallel mechanisms has been carried out [22, 35-37]. Recently, Pashkevich [23-24, 38] propose a virtual joint method (VJM) to formulate the stiffness models of parallel mechanisms, in which 6-DoF virtual springs are employed to describe the link and joint deformations.

In consequence, it should be pointed out that the semi-analytical approach based on virtual work principle is more suitable for the parameterized stiffness analysis, estimation and design comparing to the other two approaches, as it combines the accuracy of numerical methods and explicitness of analytical method. From the viewpoint of topology structure, the novel T5 PKM consists of two substructures in terms of the redundant and overconstrained parallel mechanisms that are connected by two interlinked revolute joints. Hence the deformations stemmed from both redundant substructure and overconstrained substructure are interactive by the two interlinked revolute joints. The above-mentioned semi-analytical approach cannot apply directly to the stiffness modeling of T5 PKM because of its unique structure. How to deal with the deformation contribution of interlinked revolute joints to the substructures is of vital important. In addition, the Jacobian matrix is a key issue in the stiffness modeling of parallel mechanism utilizing the semi-analytical approach, yet how to apply Jacobian matrix to the stiffness model of the redundant and overconstrained parallel mechanisms still remains an open problem.

Moreover, for the 5-DoF PKMs with horizontal layout, the deformation caused by the gravitational forces of the machine should not be neglected in the stiffness model since it will has great influence on the deformations of the end reference point. However, the stiffness model considering gravitational effects is an open issue to be investigated although several attempts have been made to deal with this problem [24, 38-40]. In addition, how to verify the validity of the stiffness model considering gravitational effects is also a challenging problem.

This paper formulates a semi-analytical stiffness model of T5 PKM considering gravitational effects, and verifies its validity by means of the stiffness experiment. Having outlined the existing approaches and challenges in Section 1, this paper is organized as follows. The system description and inverse position analysis are addressed briefly in Section 2. Section 3 formulates the stiffness model of T5 PKM, in which the stiffness models of substructure I and II are obtained in Section 4. The stiffness experiment is carried out in Section 5 to illustrate the validity of this stiffness model. Section 6 demonstrates the stiffness analysis in the prescribed workspace and the gravitational effects to T5 PKM before the conclusions are drawn in Section 7.

\section{T5 PKM description and inverse position analysis}

T5 PKM is composed of a fixed base, a moving platform, a platform I, a UP limb, five UPS limbs, two interlinked R joints denoted by $\mathrm{IR}_{1}$ and $\mathrm{IR}_{2}$, closed-loop I and closed-loop II. As shown in Fig. 1, The five UPS limbs connect the fixed base by U joints and the 1st and 2nd, 3rd and 4th UPSS limbs join platform I together by $\mathrm{IR}_{1}$ and $\mathrm{IR}_{2}$, respectively, while the 5th UPS limb links platform I by $\mathrm{S}$ joint. The UP limb joins the fixed base by U joint with its center denoted by point $O$ and connects rigidly to platform I at point $A_{6}$. The prescribed position workspace of T5 PKM is considered as a cylinder whose radius is $R$ and height is $h$, and the distance between 
point $O$ and the upper surface of the prescribed workspace is represented by $H$. As is shown in Fig.1(b), point $B_{i}$ and $A_{i}$ denote centers of $\mathrm{U}$ joint and $\mathrm{S}$ joint of the $i$ th UPS limb $(i=1 \sim 5)$, respectively. The circumradiuses of the fixed base and the platform I are denoted by $b$ and $a$, respectively. The point $B_{12}, B_{34}$ are the midpoints of line $B_{1} B_{2}$ and $B_{3} B_{4}$ which are perpendicular to each other, and the distance between $B_{12}$ (or $\left.B_{34}\right)$ to $B_{j}(j=1 \sim 4)$ is represented by $b_{0}$. Similarly, point $A_{12}, A_{34}$ are the midpoints of line $A_{1} A_{2}$ and $A_{3} A_{4}$ which are also mutually perpendicular, and the distance between $A_{12}$ (or $\left.A_{34}\right)$ to $A_{j}(j=1 \sim 4)$ is deboted by $h_{0}$.

In order to describe the motion in a distinct way, T5 PKM can be divided into two parallel mechanims in terms of a 3-DoF redundant parallel mechanisms and a 2-DoF overconstrained parallel mechanism [19]. Topology strucutre of the 3-DoF redundant parallel mechanisms is described by the fixed base, platform I, five UPS limbs, $I R_{1}, I_{2}$ and UP limb, which is named as substructure I in this paper. The 2-DoF overconstrained parallel mechanism is made up of two parallelogram-based closed-loop I and II with topology RUUR, which is called after substructure II in this paper.

As shown in Fig. 2, in the closed-loop I and II, one axis of the $U$ joint is collinear with that of the other $U$ joint, and the other two axes are parallel to the $\mathrm{R}$ joints and to those of $\mathrm{IR}_{1}$ and $\mathrm{IR}_{2}$, respectively. Point $E_{1}, E_{2}, F_{1}$ and $F_{2}$ denote the centers of $\mathrm{R}$ joints, point $E_{3}, E_{4}, F_{3}$ and $F_{4}$ denote the centers of $\mathrm{U}$ joints, respectively. The output link $E_{3} E_{4}$ and $F_{3} F_{4}$ of closed-loop I and II are articulated by one $\mathrm{R}$ joint with its center represented by point $D$, in which the output link $E_{3} E_{4}$ is designed as the moving platform of the substructure II and T5 PKM shown in Fig. 1(a). What should bear in mind is that T5 PKM is not a hybrid mechanism simply added by substructure I and II, the inputs of the substructure II are given by the $\mathrm{IR}_{1}$ and $\mathrm{IR}_{2}$ of substructure $\mathrm{I}$. In consequence, T5 PKM is a parallel mechanism form the viewpoint of the topology structure, which is essentially different from Tricept-like and Sprint Z3 Head-like PKMs.

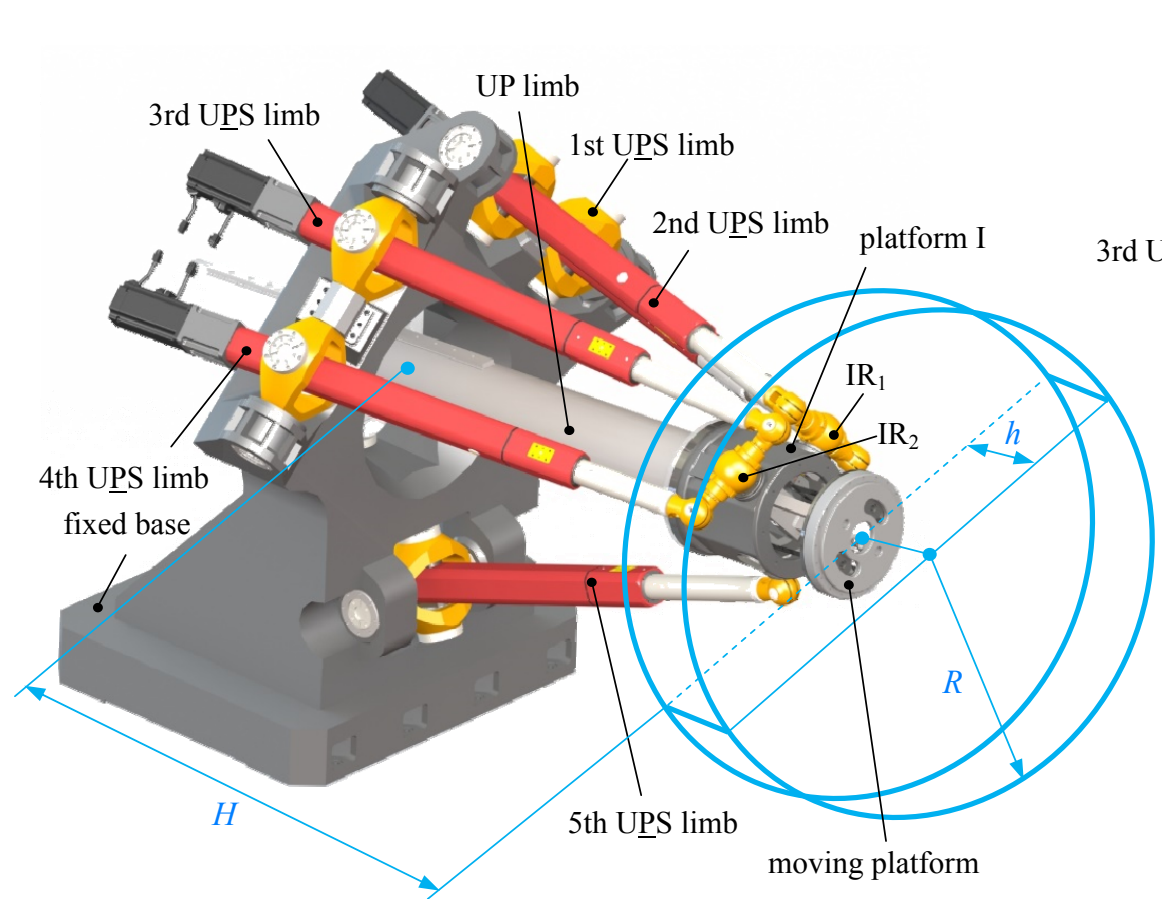

(a)

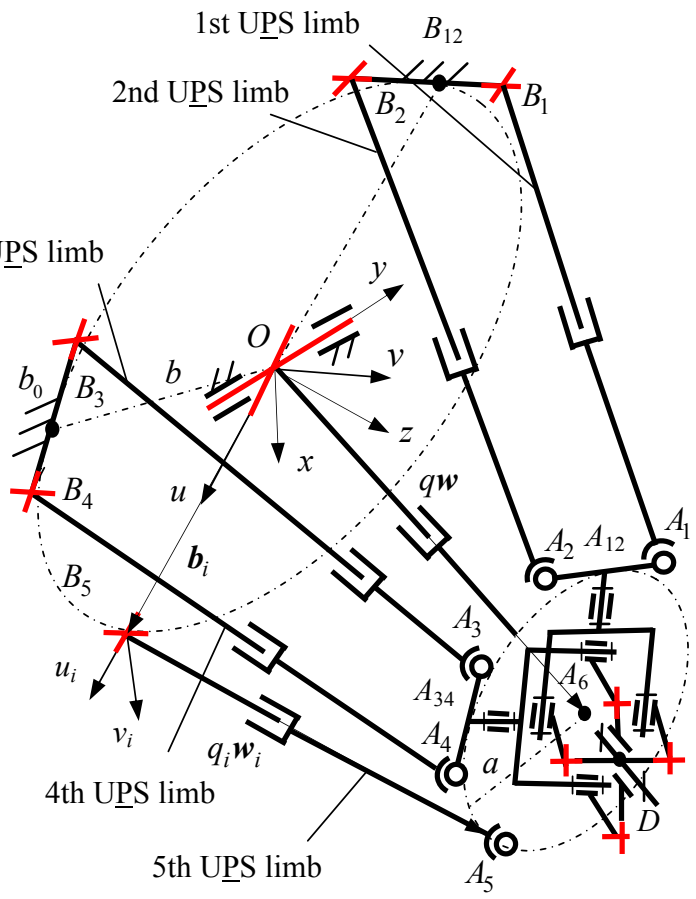

(b)

Fig.1 (a) 3-D model and (b) schematic diagram of T5 PKM

In order to describe the motions of T5 PKM, several reference frames are established. As shown in Fig. 1(b), a fix reference frame 
designated as frame $O-x y z$ is fixed to the center point $O$ with the $z$-axis normal to the fixed base and the $y$-axis coincident with the proximal axis of central $\mathrm{U}$ joint. As shown in Fig. 2, a moving reference frame $A_{6}-u v w$ is assigned to the center point $A_{6}$ of platform I. Its $u$-axis is parallel to the distal axis of central $\mathrm{U}$ joint and the $w$-axis coincides with the axis of $\mathrm{P}$ joint of the UP limb. Reference frames $B_{i}-u_{i} v_{i} w_{i}(i=1 \sim 5)$ shown in Fig. 1(b) are defined at point $B_{i}$ to describe the configuration of UPS limbs, its $u_{i}$-axis is collinear with the distal axis of the $i$ th $\mathrm{U}$ joint while $w_{i}$ coincides with the axis of $\underline{P}$ joint of the $i$ th UPS limb. By rotating frame $A_{6}-u v w$ about the $w$-axis with $\psi(\psi=-5 \pi / 4)$, the reference frame $A_{6}-u^{\prime} v^{\prime} w^{\prime}$ is established at point $A_{6}$. As shown in Fig. 2, a reference frame $D-\bar{u}_{1} \bar{v}_{1} \bar{w}_{1}$ is set at point $D$, whose $\bar{u}_{1}$-axis is parallel to line $E_{3} E_{4}$ and the $\bar{v}_{1}$-axis is consistent with the $v^{\prime}$-axis. Similarly, a reference frame $D-\bar{u}_{2} \bar{v}_{2} \bar{w}_{2}$ is located at point $D$, whose $\bar{u}_{2}$-axis is collinear with the $u^{\prime}$-axis and the $\bar{v}_{2}$-axis is parallel to line $F_{3} F_{4}$. In addition, a moving reference frame $D-u^{\prime \prime} v^{\prime \prime} w^{\prime \prime}$ is defined with its $u^{\prime \prime}$-axis parallel to line $E_{3} E_{4}$ and the $w^{\prime \prime}$-axis normal to the plane of the moving platform.

Based upon aforementioned definitions, the orientation matrix $\boldsymbol{R}$ of frame $A_{6}-u v w$ with respect to frame $O-x y z$ can be described by rotating about $y$-axis with $\alpha$ angle and $u$-axis with $\beta$ angle successively. Such that, the position vector, $\boldsymbol{r}=\left(\begin{array}{lll}x & y & z\end{array}\right)^{\mathrm{T}}$, of point $D$ in frame $O-x y z$ can be expressed by

$$
\boldsymbol{r}=(q+d) \boldsymbol{w}
$$

where

$$
q \boldsymbol{w}=\boldsymbol{b}_{i}+q_{i} \boldsymbol{w}_{i}-\boldsymbol{a}_{i}, \quad i=1,2, \cdots, 5
$$

herein $q$ and $\boldsymbol{w}$ are the length and unit vector of UP limb, resepectively, $\boldsymbol{b}_{i}$ and $\boldsymbol{a}_{i}$ represent vectors of point $B_{i}$ and $A_{i}$ in frame $O-x y z$, $q_{i}$ and $\boldsymbol{w}_{i}$ are the length and unit vector of the $i$ th UPS limb, $d$ denotes the distance from point $A_{6}$ to point $D$,

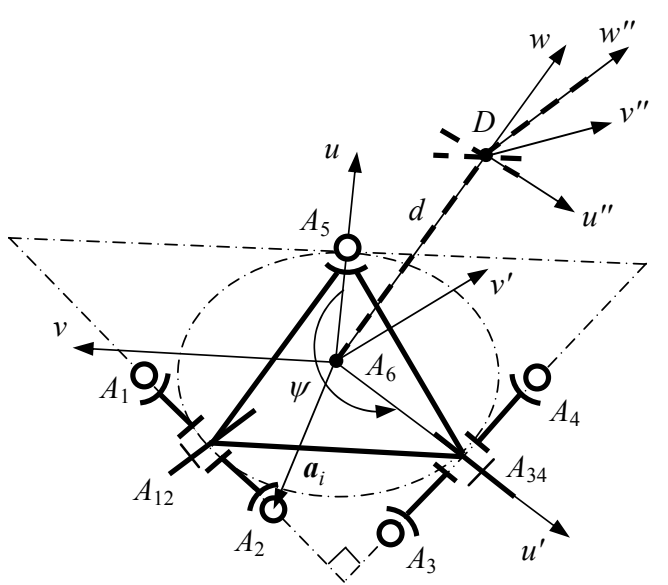

(a)

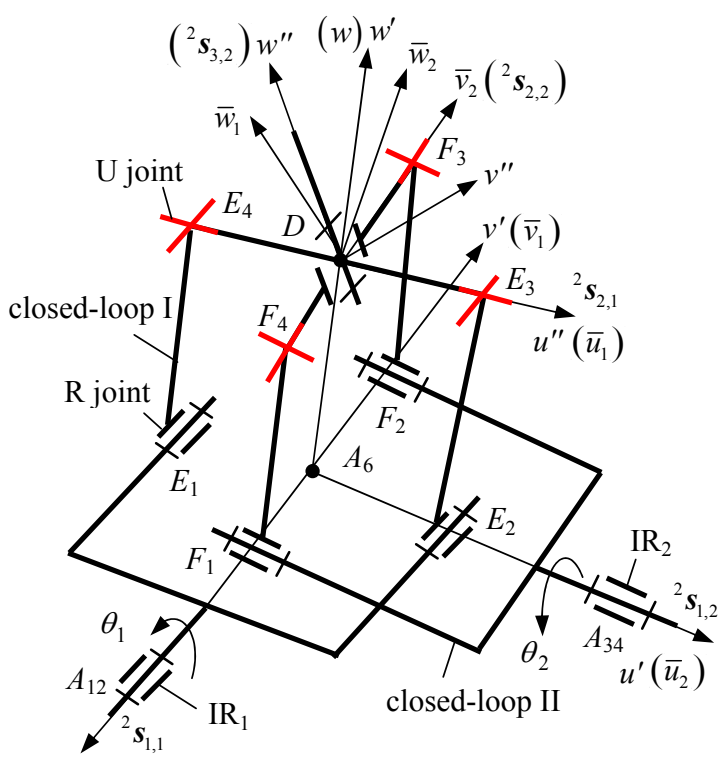

(b)

Fig.2 (a) Description of platform I in a plane and (b) schematic diagram of substructure II 


$$
\boldsymbol{a}_{i}= \begin{cases}\boldsymbol{R}\left(\boldsymbol{a}_{10}+\boldsymbol{R}_{\psi} \boldsymbol{R}_{\theta_{1}} \boldsymbol{h}_{i}\right), & i=1,2 \\ \boldsymbol{R}\left(\boldsymbol{a}_{20}+\boldsymbol{R}_{\psi} \boldsymbol{R}_{\theta_{2}} \boldsymbol{h}_{i}\right), & i=3,4 \\ \boldsymbol{R} \boldsymbol{a}_{30}, & i=5\end{cases}
$$

where $\boldsymbol{a}_{10}, \boldsymbol{a}_{20}, \boldsymbol{a}_{30}$ are the vectors of point $A_{6}$ to point $A_{12}$, point $A_{34}$, point $A_{5}$ in frame $A_{6}-u v w$, respectively. $\boldsymbol{R}_{\psi}$ is the orientation matrix of frame $A_{6}-u^{\prime} v^{\prime} w^{\prime}$ with respect to frame $A_{6}-u v w . \boldsymbol{R}_{\theta_{1}}$ and $\boldsymbol{R}_{\theta_{2}}$ are the orientation matrixes of $\mathrm{IR}_{1}$ and $\mathrm{IR}_{2}$ rotating about $v^{\prime}$-axis and $u^{\prime}$-axis with $\theta_{1}$ and $\theta_{2}$, respectively. It is worth to mention that $\boldsymbol{R}_{\theta_{1}}$ and $\boldsymbol{R}_{\theta_{2}}$ are the orientation matrixes of frame $D-\bar{u}_{1} \bar{v}_{1} \bar{w}_{1}$ and frame $D-\bar{u}_{2} \bar{v}_{2} \bar{w}_{2}$ respect to frame $A_{6}-u^{\prime} v^{\prime} w^{\prime}$, respectively. $\boldsymbol{h}_{i}$ (when $i=1,2, \cdots, 4$ ) is the vector from point $A_{12}$ or point $A_{34}$ to $A_{i}$ in frame $A_{6}-u v w$.

The orientation matrix of frame $D-u^{\prime \prime} v^{\prime \prime} w^{\prime \prime} \quad$ with respect to frame $A_{6}-u^{\prime} v^{\prime} w^{\prime} \quad$ is calculated as

$$
\boldsymbol{R}_{\theta}=\left[\begin{array}{lll}
\boldsymbol{u}^{\prime \prime} & \boldsymbol{v}^{\prime \prime} & \boldsymbol{w}^{\prime \prime}
\end{array}\right]
$$

where $\boldsymbol{u}^{\prime \prime}=\operatorname{Rot}\left(\boldsymbol{v}^{\prime}, \theta_{1}\right) \boldsymbol{u}^{\prime}, \boldsymbol{w}^{\prime \prime}=\left[\operatorname{Rot}\left(v^{\prime}, \theta_{1}\right) \boldsymbol{u}^{\prime}\right] \times\left[\operatorname{Rot}\left(u^{\prime}, \theta_{2}\right) \boldsymbol{v}^{\prime}\right]$ and $\boldsymbol{v}^{\prime \prime}=\boldsymbol{w}^{\prime \prime} \times \boldsymbol{u}^{\prime \prime}$.

Supposed that the position vector $\boldsymbol{r}$ of point $D$ in frame $O-x y z$ and the angles $\varphi_{x}$ and $\varphi_{y}$ achieved by rotating the moving platform about $x$ and $y$ axis are known, and then the vector $\boldsymbol{w}$ can be determined by Eq. (1) and the unit vector $\boldsymbol{w}_{6}$ of the spindle axis whose direction is perpendicular to the plane of the moving platform in frame $O-x y z$ can be expressed.

Based upon Eq. (1), $\alpha$ and $\beta$ can be calculated easily and then the orientation matrix $\boldsymbol{R}$ of frame $A_{6}-u v w$ with respect to frame $O-x y z$ is formulated. Then the orientation matrixes $\boldsymbol{R}_{\theta_{1}}$ and $\boldsymbol{R}_{\theta_{2}}$ are formulated by

$$
\boldsymbol{w}^{\prime \prime}=\boldsymbol{R}_{\psi}^{\mathrm{T}} \boldsymbol{R}^{\mathrm{T}} \boldsymbol{w}_{6}=\left(\begin{array}{lll}
w_{x}^{\prime \prime} & w_{y}^{\prime \prime} & w_{z}^{\prime \prime}
\end{array}\right)^{\mathrm{T}}
$$

herein, it is noted that $\theta_{1}=\arctan \left(\frac{w_{x}^{\prime \prime}}{w_{z}^{\prime \prime}}\right)$ and $\theta_{2}=\arctan \left(-\frac{w_{y}^{\prime \prime}}{w_{z}^{\prime \prime}}\right)$.

Therefore, the inverse position problem of T5 PKM, i.e. calculation of $q_{i}$ is solved by means of Eq. (1) after determining $\boldsymbol{a}_{i}$ according to the values of $\theta_{1}$ and $\theta_{2}$.

\section{Stiffness modeling of T5 PKM considering gravitational effects}

Based upon the analysis results of Section 2, T5 PKM can be divided into substructure I and II in terms of the 3-DoF redundant parallel mechanism and the 2-DoF overconstrained parallel mechanism, in which $\mathrm{IR}_{1}$ and $\mathrm{IR}_{2}$ shown in Fig. 1 and 2 are included by the two substructures in common. The $\mathrm{IR}_{1}$ and $\mathrm{IR}_{2}$ are passive joints in the substructure $\mathrm{I}$ while they are actuated joints in the substructure II. Therefore, the deformation of T5 PKM can be calculated by the sum of the deformations of the two substructures in the light of the deformation superposition principle, in which the deformation contributions of $\mathrm{IR}_{1}$ and $\mathrm{IR}_{2}$ to the two substructures respectively must be considered, however the compliance calculation of $\mathrm{IR}_{1}$ and $\mathrm{IR}_{2}$ is an important and challenging issue. In addition, the component gravities of T5 PKM are considered as the external payloads exerting on the moving platform of T5 PKM in this paper. This treatment will be verified by the stiffness experiment of T5 PKM. 




(a)



(b)

Fig.3 Free-body diagrams of (a) substructure I and (b) substructure II

On the basis of screw theory, the stiffness modeling flow of T5 PKM can be concluded in this paper as: 1) the wrench and twist mapping models of substructure I and substructure II are formulated considering gravitational effects and then the compliance models in their joint space are obtained, respectively; 2) utilizing virtual work principle and considering deformation compatibility conditions, the compliance models of the two substructures in their operated space are established by means of Jacobian matrix; 3) based upon deformation superposition principle, the stiffness model of T5 PKM are formulated considering the deformation contributions of IR $_{1}$ and $\mathrm{IR}_{2}$.

\subsection{Wrench mapping model}

The wrenches exerting on T5 PKM are considered as the external payload, internal payload of actuations/constraints and gravity. As shown in Fig. 3(a), the equation of static equilibrium of substructure I at point $A_{6}$ can be written as

$$
{ }^{1} \mathbf{S}_{w, E}+{ }^{1} \mathbf{S}_{w, G}=\sum_{k a=1}^{5}{ }^{1} f_{a, k a}{ }^{1} \hat{\mathbf{S}}_{w a, k a}-\sum_{j a=1}^{2}{ }^{1} \tau_{I R_{j a}}{ }^{1} \hat{\mathbf{S}}_{w a, I R R_{j a}}+\sum_{k c=1}^{3}{ }^{1} f_{c, k c}{ }^{1} \hat{\mathbf{S}}_{w c, k c}, \quad k a=1,2, \cdots, 5, j a=1,2, \quad k c=1,2,3
$$

where ${ }^{1} \$_{w, E}$ represents the external wrench applying on point $A_{6},{ }^{1} \mathbf{S}_{w, G}$ is the equivalent gravitational wrench of components applying on point $A_{6},{ }^{1} \hat{\boldsymbol{\$}}_{w a, k a}$ and ${ }^{1} f_{a, k a}$ represent the unit wrench of permission and its intensity of the $k a$ th $\underline{U P S}^{\text {limb, }}{ }^{1} \hat{\boldsymbol{S}}_{w a, I R_{j a}}$ and ${ }^{1} \tau_{I R_{j a}}$ are unit wrenches and intensities of $\mathrm{IR}_{1}$ or $\mathrm{IR}_{2},{ }^{1} \hat{\boldsymbol{S}}_{w c, k c}$ and ${ }^{1} f_{c, k c}$ denote unit wrenches of constraints and their intensities of the passive UP limb, and

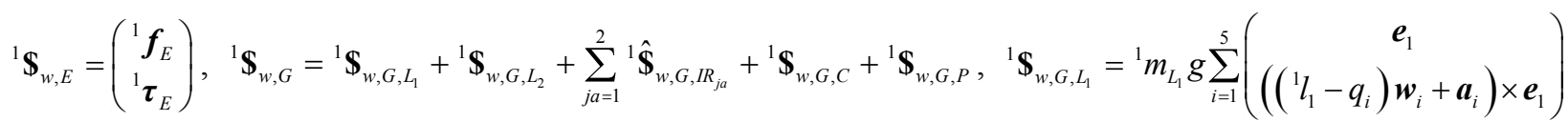

$$
\begin{aligned}
& { }^{1} \mathbf{S}_{w, G, L_{2}}={ }^{1} m_{L_{2}} g \sum_{i=1}^{5}\left(\left(\begin{array}{c}
\boldsymbol{e}_{1} \\
\left.{ }^{1} l_{2} \boldsymbol{w}_{i}+\boldsymbol{a}_{i}\right) \times \boldsymbol{e}_{1}
\end{array}\right),{ }^{1} \hat{\mathbf{S}}_{w, G, I R_{j a}}={ }^{1} m_{I R_{j a}} g\left(\begin{array}{c}
\boldsymbol{e}_{1} \\
\boldsymbol{a}_{i 0} \times \boldsymbol{e}_{1}
\end{array}\right),{ }^{1} \mathbf{S}_{w, G, C}=\rho A_{C} q g\left(\begin{array}{c}
\boldsymbol{e}_{1} \\
-\frac{1}{2} q \boldsymbol{w} \times \boldsymbol{e}_{1}
\end{array}\right)\right.
\end{aligned}
$$




$$
{ }^{1} \mathbf{S}_{w, G, P}={ }^{1} m_{P}\left(\begin{array}{c}
\boldsymbol{e}_{1} \\
-{ }^{1} l_{P} \boldsymbol{w} \times \boldsymbol{e}_{1}
\end{array}\right),{ }^{1} \hat{\boldsymbol{S}}_{w a, k a}=\left(\begin{array}{c}
\boldsymbol{w}_{i} \\
\boldsymbol{a}_{i} \times \boldsymbol{w}_{i}
\end{array}\right),{ }^{1} \hat{\boldsymbol{S}}_{w a, R, j a}=\left(\begin{array}{c}
\mathbf{0} \\
{ }^{1} \boldsymbol{\tau}_{R, j a}
\end{array}\right),{ }^{1} \hat{\boldsymbol{\$}}_{w c, 1}=\left(\begin{array}{c}
\boldsymbol{u} \\
-q \boldsymbol{w} \times \boldsymbol{u}
\end{array}\right),{ }^{1} \hat{\boldsymbol{\$}}_{w c, 2}=\left(\begin{array}{c}
\boldsymbol{v} \\
-q \boldsymbol{w} \times \boldsymbol{v}
\end{array}\right),{ }^{1} \hat{\boldsymbol{\$}}_{w c, 3}=\left(\begin{array}{c}
\mathbf{0} \\
\boldsymbol{\tau}_{w}
\end{array}\right)
$$

where ${ }^{1} \boldsymbol{f}_{E}$ and ${ }^{1} \boldsymbol{\tau}_{E}$ denote the external force and torque applying on point $A_{6},{ }^{1} \mathbf{S}_{w, G, L_{1}},{ }^{1} \mathbf{S}_{w, G, L_{2}},{ }^{1} \hat{\mathbf{S}}_{w, G, I R_{j a}},{ }^{1} \mathbf{S}_{w, G, C}$ and ${ }^{1} \mathbf{S}_{w, G, P}$ are equivalent gravitational wrenches of outer tube of the $i$ th UPS limb, inner telescopic link of the $i$ th UPS limb, $\operatorname{IR}_{1}$ or $\mathrm{IR}_{2}$, central pipe of UP limb and platform I exerting on point $A_{6}$, respectively. ${ }^{1} m_{L_{1}}$ is the mass of the outer tube, $\boldsymbol{e}_{1}$ denotes the unit vector of gravitational acceleration, and ${ }^{1} l_{1}$ represents the distance between the mass center of the outer tube and that of $\mathrm{U}$ joint. ${ }^{1} m_{L_{2}}$ denotes the mass of the inner telescopic link, and ${ }^{1} l_{2}$ represents the distance from the mass center of the inner telescopic to that of $\mathrm{S}$ joint. ${ }^{1} m_{I R_{i j}}$ is the mass of $\mathrm{IR}_{1}$ or $\mathrm{IR}_{2}$. Besides, $\rho$ and $A_{C}$ are density and sectional area of the center pipe of UP limb, ${ }^{1} m_{P}$ and ${ }^{1} l_{P}$ are the mass of platform I and the distance between the mass center of platform I to point $A_{6}$, respectively.

In a similar way, the equation of static equilibrium of substructure II shown in Fig. 3(b) is formulated as

$$
{ }^{2} \boldsymbol{\$}_{w, E}+{ }^{2} \boldsymbol{\$}_{w, G}=\sum_{k a=1}^{2}{ }^{2} \tau_{a, k a}{ }^{2} \hat{\boldsymbol{S}}_{w a, k a}+\sum_{k c c=1}^{4}{ }^{2} f_{c, k c c}{ }^{2} \hat{\boldsymbol{S}}_{w c, k c c}+\sum_{k c r=1}^{3}{ }^{2} f_{c, k c r}{ }^{2} \hat{\boldsymbol{S}}_{w c, k c r}
$$

where ${ }^{2} \boldsymbol{\$}_{w, E}$ denotes the external wrench of substructure II applying on point $D,{ }^{2} \boldsymbol{\$}_{w, G}$ is the equivalent gravitational wrench of substructure II exerting at point $D .{ }^{2} \hat{\boldsymbol{S}}_{w a, k a}$ and ${ }^{2} \tau_{a, k a}$ represent the unit wrench of actuation and its intensity, respectively. ${ }^{2} \hat{\boldsymbol{S}}_{w c, k c c}$ and ${ }^{2} f_{c, k c c}$ denote the unit wrench of constraint and its intensity, ${ }^{2} \hat{\boldsymbol{S}}_{w c, k c r}$ and ${ }^{2} f_{c, k c r}$ represent the unit wrench and intensity of over-constraints of substructure II. And

$$
\begin{aligned}
& { }^{2} \boldsymbol{\$}_{w, E}=\left(\begin{array}{c}
{ }^{2} \boldsymbol{f}_{E} \\
{ }^{2} \boldsymbol{\tau}_{E}
\end{array}\right),{ }^{2} \mathbf{S}_{w, G}=\sum_{j=1}^{2} \sum_{i=1}^{3}{ }^{2} \boldsymbol{\$}_{w, G, i, j},{ }^{2} \boldsymbol{\$}_{w, G, 1, j}={ }^{2} m_{1, j} g\left(\begin{array}{c}
\boldsymbol{e}_{1} \\
-\left({ }^{2} \boldsymbol{l}_{1, j}+d \boldsymbol{w}^{\prime}\right) \times \boldsymbol{e}_{1}
\end{array}\right) \\
& { }^{2} \boldsymbol{\$}_{w, G, 2, j}=2^{2} m_{2, j} g\left(\left(\begin{array}{c}
\boldsymbol{e}_{1} \\
\left.{ }^{2} \boldsymbol{l}_{2, j}-\frac{1}{2} d \boldsymbol{w}^{\prime}\right) \times \boldsymbol{e}_{1}
\end{array}\right),{ }^{2} \boldsymbol{\$}_{w, G, 3, j}={ }^{2} m_{3, j} g\left(\begin{array}{c}
\boldsymbol{e}_{1} \\
{ }^{2} \boldsymbol{l}_{3, j} \times \boldsymbol{e}_{1}
\end{array}\right),{ }^{2} \hat{\boldsymbol{\$}}_{w a, 1}=\left(\begin{array}{c}
\mathbf{0} \\
{ }^{2} \boldsymbol{s}_{1,1}
\end{array}\right)\right.
\end{aligned}
$$

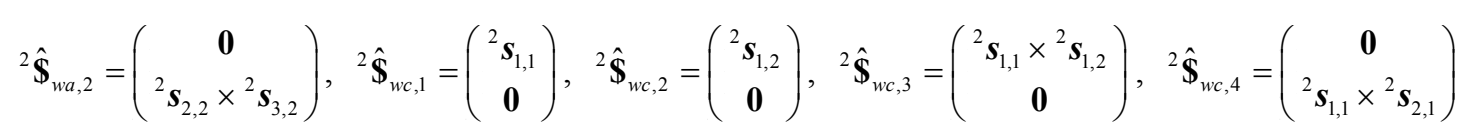

herein, ${ }^{2} \boldsymbol{f}_{E}$ and ${ }^{2} \boldsymbol{\tau}_{E}$ are external force and torque applying on point $D,{ }^{2} \boldsymbol{\$}_{w, G, i, j}$ is the equivalent gravitational wrench of $i$ th component exerting on point $D .{ }^{2} m_{i, 1}$ represents the mass of link $E_{1} E_{2}$, link $E_{1} E_{4}\left(E_{2} E_{3}\right)$, and output link $E_{3} E_{4}$ of closed-loop I, respectively. Similarly, ${ }^{2} m_{i, 2}$ is the mass of link $F_{1} F_{2}$, link $F_{1} F_{4} \quad\left(F_{2} F_{3}\right)$ and output link $F_{3} F_{4}$ of closed-loop II, respectively. ${ }^{2} \boldsymbol{I}_{1, j}$ is the vector from mass center of link $E_{1} E_{2}$ to point $A_{6}$, and ${ }^{2} \boldsymbol{l}_{2, j}$ is the vector from mass center of $E_{1} E_{4}\left(E_{2} E_{3}\right)$ or $F_{1} F_{4}\left(F_{2} F_{3}\right)$ to point $D .{ }^{2} \boldsymbol{l}_{3, j}$ denotes the vector between mass center of output link $E_{3} E_{4} \quad\left(F_{3} F_{4}\right)$ and point $D$, and ${ }^{2} \boldsymbol{s}_{i, j}$ represents the unit axis vector of the 1-DoF joints in substructure II shown in Fig. 2(b).

Based upon above-mentioned analysis, it is obvious that ${ }^{1} \boldsymbol{\tau}_{R, j a}$ and ${ }^{2} \boldsymbol{\tau}_{a, k a}$ are mutually action and reaction force, and then the 
wrench model of T5 PKM are calculated as

$$
\mathbf{\$}_{w}=\boldsymbol{T}^{-\mathrm{T} 1} \mathbf{\$}_{w}+{ }^{2} \mathbf{\$}_{w}
$$

where $\boldsymbol{\$}_{w}=\mathbf{\$}_{w, E}+\mathbf{\$}_{w, G},{ }^{1} \boldsymbol{\$}_{w}={ }^{1} \boldsymbol{\$}_{w, E}+{ }^{1} \boldsymbol{\$}_{w, G},{ }^{2} \boldsymbol{\$}_{w}={ }^{2} \boldsymbol{\$}_{w, E}+{ }^{2} \boldsymbol{\$}_{w, G}$ and $\boldsymbol{T}=\left[\begin{array}{cc}\boldsymbol{E}_{3} & -d[\boldsymbol{w} \times] \\ \mathbf{0}_{3 \times 3} & \boldsymbol{E}_{3}\end{array}\right] . \boldsymbol{E}_{3}$ denotes $3 \times 3$ unit matrix while $[\boldsymbol{w} \times]$ represents the skew-symmetric matrix relating to vector $\boldsymbol{w}$.

\subsection{Twist Mapping Model}

As shown in [19], the twist $\boldsymbol{\$}_{t}$ of T5 PKM with respect to reference point $D$ is the liner superposition of twists ${ }^{1} \boldsymbol{\$}_{t}$ and ${ }^{2} \boldsymbol{\$}_{t}$ produced by substructure I and II respectively, which is

$$
\mathbf{\$}_{t}=\boldsymbol{T}^{1} \mathbf{\$}_{t}+{ }^{2} \mathbf{\$}_{t}
$$

Based upon the wrench and twist mapping models, the compliance model of T5 PKM can be formulated as follows in the light of Hooke's Law.

$$
C \$_{w}=\$_{t}
$$

herein $\boldsymbol{C}$ denotes the compliance matrix of T5 PKM.

Substituting Eq. (7) and Eq. (8) into Eq. (9), $\boldsymbol{C}$ can be expressed as

$$
\boldsymbol{C}=\boldsymbol{T}^{1} \boldsymbol{C} \boldsymbol{T}^{\mathrm{T}}+{ }^{2} \boldsymbol{C}
$$

where ${ }^{1} \boldsymbol{C}^{1} \mathbf{S}_{w}={ }^{1} \mathbf{S}_{t}$ and ${ }^{2} \boldsymbol{C}^{2} \boldsymbol{S}_{w}={ }^{2} \boldsymbol{S}_{t} .{ }^{1} \boldsymbol{C}$ and ${ }^{2} \boldsymbol{C}$ are compliance matrixes of substructure I and II with respect to point $A_{6}$ and $D$, respectively.

It is concluded from aforementioned compliance/stiffness modeling of T5 PKM that: 1) the gravities of two substructures are equivalent to external payloads exerting on point $A_{6}$ and point $D$ by means of static equilibrium, respectively; 2) the wrench and twist models of two substructures are the same as those presented in [19] since the gravities are regarded as the external payloads; 3) the stiffness matrix of T5 PKM can be obtained after computing ${ }^{1} \boldsymbol{C}$ and ${ }^{2} \boldsymbol{C}$ in Section 4, in which the contribution of $\mathrm{IR}_{1}$ and $\mathrm{IR}_{2}$ should be considered.

Concerning Eq. (10), several assumptions should be made as: 1) the friction and contact deformation within the joints are ignored; 2) the deformations of the components satisfy the linear superposition principle; 3) the platform I, output links $E_{3} E_{4}$ and $F_{3} F_{4}$ are regarded as rigid bodies without deformations.

\section{Stiffness models of $I R_{1}, I R_{2}$, substructure I and II}

\section{1 $I R_{1}$ and $I R_{2}$}

As shown in Fig. 4, the structures of $\mathrm{IR}_{1}$ and $\mathrm{IR}_{2}$ look like the capital letter ' $\mathrm{T}$ ', the horizontal parts link to the 1 st and $2 \mathrm{nd}$ (or 3rd and 4th) UPS limbs at point $A_{k}$ and $A_{k+1}(k=1,3)$, respectively, while the vertical part connects to platform I by rotational components at point $A_{k, k+1}^{\prime}$ and joins actuated joints of substructure II at point $A_{k, k+1}^{\prime \prime}$ rigidly. Based upon aforementioned analysis, 
it is reasonable to know that the axial forces ${ }^{1} \boldsymbol{f}_{a, k}$ and ${ }^{1} \boldsymbol{f}_{a, k+1}$ caused by UPS limbs applies on the horizontal part of $\mathrm{IR}_{1}$ and $\mathrm{IR}_{2}$, while the force ${ }^{1} \boldsymbol{f}_{p, k r}$ and torque ${ }^{1} \boldsymbol{\tau}_{p, k r}$ caused by platform I exert on point $A_{k, k+1}^{\prime}$. The torque $-{ }^{2} \boldsymbol{\tau}_{a, k r}$ (equal to ${ }^{1} \boldsymbol{\tau}_{R, k r}$ ) caused by substructure II exerts on point $A_{k, k+1}^{\prime \prime}$, whose direction is the same as the rotational axis of $\mathrm{IR}_{1}$ and $\mathrm{IR}_{2}$.

As shown in Fig. 4, the deformations of $\mathrm{IR}_{1}$ and $\mathrm{IR}_{2}$ can be divided into the rigid body motion and the elastic deformation. The initial configuration of $\mathrm{IR}_{1}$ and $\mathrm{IR}_{2}$ is represented by state 1 , then they move rigidly to state 2 . Supposed that UPS limbs stay still while $I R_{1}$ and $I_{2}$ begin to deform, which is demonstrated by the red lines in Fig. 4.

Therefore, the compliance of substructure I caused by five UPS limbs can be written as follows when considering the deformation effects of $\mathrm{IR}_{1}$ and $\mathrm{IR}_{2}$.

$$
{ }^{1} \boldsymbol{C}_{a}={ }^{1} \boldsymbol{C}_{a, 1}+\sum_{i=1}^{2}{ }^{1} \boldsymbol{C}_{a, 2, i}
$$

where ${ }^{1} \boldsymbol{C}_{a, 1}$ is the compliance matrix of substructure I at point $A_{6}$ assuming that $\mathrm{IR}_{1}$ and $\mathrm{IR}_{2}$ are treated as rigid bodies. ${ }^{1} \boldsymbol{C}_{a, 2, i}$ is the compliance matrix substructure I at point $A_{6}$ caused merely by deformation of $\mathrm{IR}_{1}$ and $\mathrm{IR}_{2}$, which can be measured by means of FEA software. It is worth mentioning that the rank of ${ }^{1} \boldsymbol{C}_{a, 2, i}$ is 5 since the angular stiffness about rotational axial of $\mathrm{IR}_{1}$ and $\mathrm{IR}_{2}$ is zero.

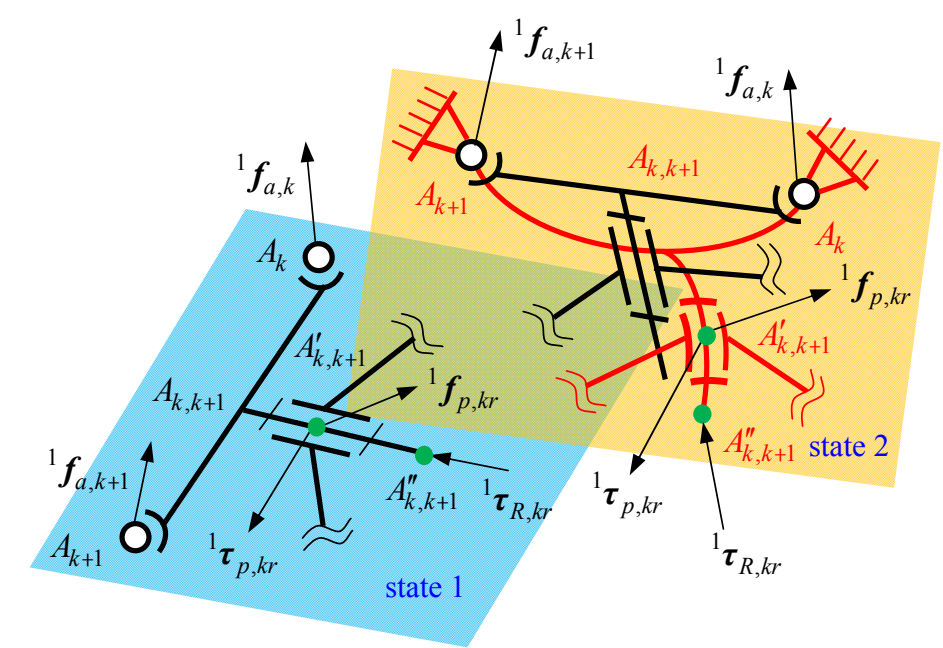

Fig. 4 Force and deformation of $\mathrm{IR}_{1}$ or $\mathrm{IR}_{2}$

When considering the deformation effects of $\mathrm{IR}_{1}$ and $\mathrm{IR}_{2}$ to actuated compliance of substructure II, its compliance matrix will be determined by the compliance matrices of $\mathrm{IR}_{1}$ and $\mathrm{IR}_{2}$ at $A_{k, k+1}^{\prime}$ and of the components between $A_{k, k+1}^{\prime}$ and $A_{k, k+1}^{\prime \prime}$, which is

$$
{ }^{2} \overline{\boldsymbol{C}}_{a c, j}={ }^{2} \overline{\boldsymbol{C}}_{a c 1, j}+{ }^{2} \overline{\boldsymbol{C}}_{a c 2, j}
$$

where ${ }^{2} \overline{\boldsymbol{C}}_{a c, j}$ is the actuated compliance matrix of closed-loop I and II. ${ }^{2} \overline{\boldsymbol{C}}_{a c 1, j}$ is the compliance matrix of $\mathrm{IR}_{1}$ and $\mathrm{IR}_{2}$ at $A_{k, k+1}^{\prime}$, which can be calculated by ${ }^{1} \boldsymbol{C}_{a, 2, i} \cdot{ }^{2} \overline{\boldsymbol{C}}_{a c 2, j}$ denotes the compliance matrix of component between $A_{k, k+1}^{\prime}$ and $A_{k, k+1}^{\prime \prime}$. Even though it is easy to get analytical result of ${ }^{2} \overline{\boldsymbol{C}}_{a c 1, j}$ and ${ }^{2} \overline{\boldsymbol{C}}_{a c 2, j}$ by multi-beam approximation, FEA software based method is applied here to obtain more reliable results. To apply this approach to determine ${ }^{2} \overline{\boldsymbol{C}}_{a c 2, j}$, platform I is fixed and the actuation wrench ${ }^{2} \boldsymbol{\$}_{w a, k a}$, the 
constrained wrench ${ }^{2} \boldsymbol{S}_{\text {wac }, k c c}$ and ${ }^{2} \boldsymbol{\$}_{\text {wack } k r}$ are applied to point $A_{k, k+1}^{\prime \prime}$, and then the corresponding linear and angular displacement can be evaluated, which are the columns of the compliance matrix ${ }^{2} \overline{\boldsymbol{C}}_{a c 2, j}$.

\subsection{Substructure I}

Based upon aforementioned analysis, six dimensional deformations of platform I at point $A_{6}$ caused by ${ }^{1} \boldsymbol{\$}_{w}$ includes tension/compression deformation along $\boldsymbol{w}_{i}$ of the $i$ th UPS limb $(i=1,2, \cdots, 5)$, five dimensional deformations of $\mathrm{IR}_{1}, \mathrm{IR}_{2}$ and bending/torsion deformation of UP limb. The twist of point $A_{6}$ can be expressed as follows in the light of linear superposition principle.

$$
{ }^{1} \mathbf{S}_{t}={ }^{1} \mathbf{S}_{t a}+{ }^{1} \mathbf{S}_{t b}+{ }^{1} \mathbf{S}_{t t}={ }^{1} \boldsymbol{C}^{1} \mathbf{S}_{w}
$$

where ${ }^{1} \mathbf{S}_{t a}$ denotes the twist resulted from UPS limbs, $\mathrm{IR}_{1}$ and $\mathrm{IR}_{2},{ }^{1} \mathbf{S}_{t b}$ and ${ }^{1} \mathbf{S}_{t t}$ represent the bending and torsional twists caused by UP limb, and ${ }^{1} \boldsymbol{C}={ }^{1} \boldsymbol{C}_{a}+{ }^{1} \boldsymbol{C}_{b}+{ }^{1} \boldsymbol{C}_{t}$.

\subsubsection{Determination of ${ }^{1} C_{a}$}

The forces applying on the five UPS limbs are merely tension/compression and it allows U모 limbs to be regarded as 1-DoF spring systems, hence the compliance of the $i$ th UPS limb at $A_{i}$ can be formulated as

$$
c_{A_{i}}=\sum_{j=1}^{7} c_{a, i, j}, \quad i=1,2, \cdots, 5, \quad j=1,2, \cdots, 7
$$

where $c_{a, i, 1}$ denotes the axial compliance of $\mathrm{S}$ joints. $c_{a, i, 2}, c_{a, i, 3}, c_{a, i, 4}, c_{a, i, 5}$ and $c_{a, i, 6}$ represent the axial compliance of $\underline{\mathrm{P}}$ joints component including inner telescopic link, screw nut, lead screw, bearings and outer tube, respectively. $c_{a, i, 7}$ denotes the axial compliance of $U$ joint.

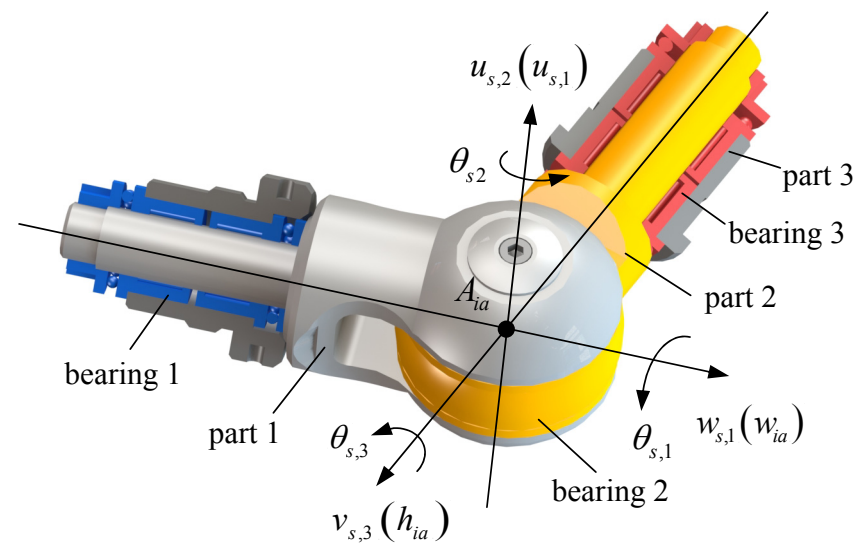

Fig. 5 3-D model of S joint in the 1st, 2nd, 3rd and 4th UPS limb

The $\mathrm{S}$ joint of UPS limbs is composed of three $\mathrm{R}$ joints whose axes are linear independent and perpendicular mutually. Taking $\mathrm{S}$ joint shown in Fig. 5 of the 1st, 2nd, 3rd and 4th UPS limb as example, the reference frames $A_{i a}-u_{s, j a} v_{s, j a} w_{s, j a} \quad(i a=1,2, \cdots, 4$, $j a=1,2,3)$ is designated to $A_{i}$, whose $w_{s, 1}$-axis is collinear with the $w_{i a}$-axis of the $i a$ th UPS limb, the $u_{s, 2}$-axis is coincident with 
that of the second R joint and the $v_{s, 3}$-axis coincides with $\boldsymbol{h}_{i a}$. Supposed that the orientation matrix of frame $A_{i a}-u_{s, j a} v_{s, j a} w_{s, j a}$ with respect to frame $A_{i a}-u_{s, j a-1} v_{s, j a-1} w_{s, j a-1}$ is $\boldsymbol{R}_{s, j a}$, and then the orientation matrix of frame $A_{i a}-u_{s, 3} v_{s, 3} w_{s, 3}$ with respect to frame $A_{i a}-u_{s, 1} v_{s, 1} w_{s, 1}$ can be expressed as

$$
\boldsymbol{R}_{s}=\boldsymbol{R}_{s, 1} \boldsymbol{R}_{s, 2} \boldsymbol{R}_{s, 3}
$$

where $\boldsymbol{R}_{s, 1}=\operatorname{Rot}\left(\boldsymbol{w}_{s, 1}, \theta_{s, 1}\right), \quad \boldsymbol{R}_{s, 2}=\operatorname{Rot}\left(\boldsymbol{u}_{s, 2}, \theta_{s, 2}\right)$ and $\boldsymbol{R}_{s, 3}=\operatorname{Rot}\left(\boldsymbol{v}_{s, 3}, \theta_{s, 3}\right) . \theta_{s, 1}, \theta_{s, 2}$ and $\theta_{s, 3}$ are obtained by means of the inverse position analysis in Section 2.

In consequence, the $3 \times 3$ linear compliance matrix of $\mathrm{S}$ joint is formulated as

$$
\boldsymbol{C}_{s}=\boldsymbol{C}_{s, 1}+\boldsymbol{C}_{s, 2}+\boldsymbol{C}_{s, 3}
$$

where $\boldsymbol{C}_{s, 1}=\boldsymbol{R}_{s, 1} \overline{\boldsymbol{C}}_{s, 1} \boldsymbol{R}_{s, 1}^{\mathrm{T}}, \boldsymbol{C}_{s, 2}=\left(\boldsymbol{R}_{s, 1} \boldsymbol{R}_{s, 2}\right) \overline{\boldsymbol{C}}_{s, 2}\left(\boldsymbol{R}_{s, 1} \boldsymbol{R}_{s, 2}\right)^{\mathrm{T}}$ and $\boldsymbol{C}_{s, 3}=\boldsymbol{R}_{s} \overline{\boldsymbol{C}}_{s, 3} \boldsymbol{R}_{s}^{\mathrm{T}}$. The linear compliance of three R joints in each reference frame are represented by $\overline{\boldsymbol{C}}_{s, 1}, \overline{\boldsymbol{C}}_{s, 2}$ and $\overline{\boldsymbol{C}}_{s, 3}$, respectively. It is noted that the third element of the diagonal of $\boldsymbol{C}_{s}$ is the axial linear compliance of $\mathrm{S}$ joint, that is $c_{a, i, 1}$.

The axial compliance of the inner telescopic link, screw nut, bearings and outer tube are constant. The lead screw is fixed on one side and free on the other side, and then its axial compliance is determined by the distance between screw nut and the fixed end as

$$
c_{a, i, 4}=\frac{q_{i}-L_{s c}}{E A_{s c}}
$$

where $L_{s c}$ is the work length, $E$ is elasticity modulus and $A_{s c}$ is sectional area of lead screw.

Similar with $\mathrm{S}$ joint, the $3 \times 3$ linear compliance matrix of $\mathrm{U}$ joint is obtained as

$$
\boldsymbol{C}_{U}=\overline{\boldsymbol{C}}_{\text {in }}+\boldsymbol{R}_{U} \overline{\boldsymbol{C}}_{\text {out }} \boldsymbol{R}_{U}^{\mathrm{T}}
$$

where $\overline{\boldsymbol{C}}_{\text {in }}$ and $\overline{\boldsymbol{C}}_{\text {out }}$ are the linear compliances of the inner and outer rotational components of $\mathrm{U}$ joint in each reference frame while $\boldsymbol{R}_{U}$ is the orientation matrix of the proximal axis with respect to the distal axis of $U$ joint. It is noted that the third element of the diagonal of $\boldsymbol{C}_{U}$ is the axial linear compliance of $\mathrm{U}$ joint, that is $c_{a, i, 7}$.

In consequence, the linear compliance of $i$ th UPS limb is achieved by substituting Eq. (15) (18) into Eq. (14), which will be mapped to point $A_{6}$ in order to formulate ${ }^{1} \boldsymbol{C}_{a}$.

In the previous work of the authors [19], the twists of UPSS limbs mapping to point $A_{6}$ can be described as

$$
\boldsymbol{J}_{x a 1}{ }^{1} \boldsymbol{\$}_{t a}=\boldsymbol{J}_{q} \boldsymbol{\Delta}_{q}
$$

where

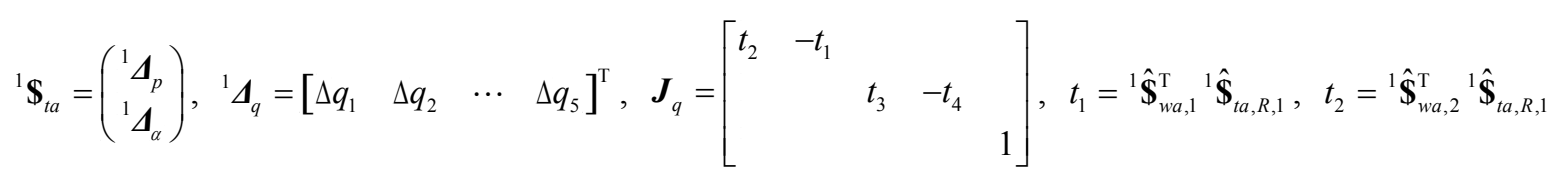




$$
t_{3}={ }^{1} \hat{\boldsymbol{S}}_{w a, 3}^{\mathrm{T}}{ }^{1} \hat{\boldsymbol{S}}_{t a, R, 2}, \quad t_{4}={ }^{1} \hat{\boldsymbol{S}}_{w a, 4}^{\mathrm{T}}{ }^{1} \hat{\boldsymbol{S}}_{t a, R, 2}, \quad \boldsymbol{J}_{x a 1}=\left[\begin{array}{ll}
\boldsymbol{J}_{x p 1} & \boldsymbol{J}_{x a 1}
\end{array}\right]=\left[\begin{array}{cc}
t_{2} \boldsymbol{w}_{1}^{\mathrm{T}}-t_{1} \boldsymbol{w}_{2}^{\mathrm{T}} & \left(\boldsymbol{a}_{1} \times t_{2} \boldsymbol{w}_{1}-\boldsymbol{a}_{2} \times t_{1} \boldsymbol{w}_{2}\right)^{\mathrm{T}} \\
t_{4} \boldsymbol{w}_{3}^{\mathrm{T}}-t_{3} \boldsymbol{w}_{4}^{\mathrm{T}} & \left(\boldsymbol{a}_{3} \times t_{4} \boldsymbol{w}_{3}-\boldsymbol{a}_{4} \times t_{3} \boldsymbol{w}_{4}\right)^{\mathrm{T}} \\
\boldsymbol{w}_{5}^{\mathrm{T}} & \left(\boldsymbol{a}_{5} \times \boldsymbol{w}_{5}\right)^{\mathrm{T}}
\end{array}\right]
$$

herein, ${ }^{1} \boldsymbol{\Delta}_{p}$ and ${ }^{1} \boldsymbol{\Delta}_{\alpha}$ represent the linear and angular deformations of platform I at point $A_{6}, \Delta q_{i}(i=1,2, \cdots, 5)$ is the linear deformation of the $i$ th UPS limb, ${ }^{1} \hat{\boldsymbol{S}}_{t a, R, 1}$ and ${ }^{1} \hat{\boldsymbol{\$}}_{t a, R, 2}$ are the unit twists of $\mathrm{IR}_{1}$ and $\mathrm{IR}_{2}$, respectively.

Taking first order perturbation on both side of Eq. (1), and resulting in

$$
{ }^{1} \boldsymbol{\Delta}_{\alpha}=\boldsymbol{T}_{\alpha p}{ }^{1} \boldsymbol{\Delta}_{p}
$$

where $\boldsymbol{T}_{\alpha p}=\frac{1}{q \boldsymbol{w}^{\mathrm{T}} \boldsymbol{w}_{e}}\left[\boldsymbol{w}_{e} \times\right]\left(\boldsymbol{E}_{3}-\boldsymbol{w} \boldsymbol{w}^{\mathrm{T}}\right), \boldsymbol{w}_{e}=\boldsymbol{u} \times \boldsymbol{e}_{2}$ and $\boldsymbol{e}_{2}=\left(\begin{array}{lll}0 & 1 & 0\end{array}\right)^{\mathrm{T}}$.

Bearing in mind the virtual work principle and Hooke's law, it is easy to formulate

$$
{ }^{1} \mathbf{S}_{t a}^{\mathrm{T} 1} \mathbf{S}_{w a}=\boldsymbol{\Delta}_{q}^{\mathrm{T} 1} \boldsymbol{f}_{a}
$$

where ${ }^{1} \boldsymbol{f}_{a}={ }^{1} \boldsymbol{K}_{a} \boldsymbol{\Delta}_{q}$ and ${ }^{1} \boldsymbol{K}_{a}=\operatorname{diag}\left(c_{A_{1}}^{-1}, c_{A_{2}}^{-1}, \cdots, c_{A_{5}}^{-1}\right)$.

When $\mathrm{IR}_{1}$ and $\mathrm{IR}_{2}$ are treated as rigid bodies without deformations, six dimensional deformations of substructure I at point $A_{6}$ merely considering the deformation contributions of UPSS limbs are formulated by substituting Eq. (19) (20) into Eq. (21) as

$$
{ }^{1} \boldsymbol{C}_{a, 1}=\boldsymbol{D}_{a}\left(\boldsymbol{J}_{a 1}^{\mathrm{T}} 1 \boldsymbol{K}_{a} \boldsymbol{J}_{a 1}\right)^{-1} \boldsymbol{D}_{a}^{\mathrm{T}}
$$

where $\boldsymbol{D}_{a}=\left[\begin{array}{ll}\boldsymbol{E}_{3} & \boldsymbol{T}_{\alpha p}^{\mathrm{T}}\end{array}\right]^{\mathrm{T}}$ and $\boldsymbol{J}_{a 1}=\boldsymbol{J}_{q}^{+}\left(\boldsymbol{J}_{x p 1}+\boldsymbol{J}_{x \alpha 1} \boldsymbol{T}_{\alpha p}\right)$.

When $\mathrm{IR}_{1}$ and $\mathrm{IR}_{2}$ are treated as elastic bodies with deformations, their stiffness matrices $\boldsymbol{K}_{I R_{1}}^{\prime}$ and $\boldsymbol{K}_{I R_{2}}^{\prime}$ are obtained by FEA software in frame $A_{k, k+1}^{\prime}-u^{\prime} v^{\prime} w^{\prime}$. Therefore, the compliance matrix of substructure I with respect to point $A_{6}$ only caused by the deformations of $\mathrm{IR}_{1}$ and $\mathrm{IR}_{2}$ can be calculated as

$$
{ }^{1} \boldsymbol{C}_{a, 2}=\left(\sum_{j=1}^{2} \boldsymbol{T}_{I R_{j}}^{-\mathrm{T}} \boldsymbol{K}_{I R_{j}}^{\prime} \boldsymbol{T}_{I R_{j}}^{-1}\right)^{-1}, j=1,2
$$

where $\boldsymbol{T}_{I R_{j}}=\left[\begin{array}{cc}\boldsymbol{R}_{I R_{j}} & {\left[\boldsymbol{a}_{I R_{j}} \times\right] \boldsymbol{R}_{I R_{j}}} \\ \mathbf{0} & \boldsymbol{R}_{I R_{j}}\end{array}\right], \boldsymbol{R}_{I R_{j}}=\boldsymbol{R} \boldsymbol{R}_{\psi} \boldsymbol{R}_{\theta_{j}}$, and $\boldsymbol{a}_{I R_{j}}=\boldsymbol{R} \boldsymbol{a}_{j 0}$.

In consequence, the compliance matrix ${ }^{1} \boldsymbol{C}_{a}$ is achieved by substituting Eq. (22) and Eq. (23) into Eq. (11).

\subsubsection{Determinations of ${ }^{1} C_{b}$ and ${ }^{1} C_{t}$}

It is concluded from Eq. (5) that the passive UP limb is exerted by the constrained wrenches that can be divided into the shearing force/bending moment along/about $u$-axis and $v$-axis and the torsional moment about $w$-axis, as shown in Fig. 6 . The aforementioned one force and two moments will lead to the bending and torsional deformations of UP limb.

As shown in Fig. 6, the P joint is regarded as the beam element because of its cylindrical structure. One side of beam element is 
named as node 1 who is also the center of $\mathrm{U}$ joint, and the other side is named as node 2 who connects rigidly to platform $\mathrm{I}$. To formulate the bending deformation of UP limb, structure matrix is used here. The dimension of the element stiffness matrix $\overline{\boldsymbol{K}}_{e}$ is $8 \times 8$ without considering the torsional deformation. The angular stiffness about $u$-axis and $v$-axis are zero, and the linear stiffness of $\mathrm{U}$ joint is $\boldsymbol{K}_{U}=\operatorname{diag}\left(k_{f u 1}, k_{f v 1}\right)$, and then the shearing force and bending moment of node 1 is $\boldsymbol{f}_{1}=\boldsymbol{K}_{U} \boldsymbol{\Delta}_{p 1}$ and $\boldsymbol{\tau}_{1}=\mathbf{0}_{2 \times 2}$, where $\boldsymbol{\Delta}_{p 1}$ denotes the linear deformation of node 1. In consequence, the stiffness matrix of node 2 is formulated as described in [34].

$$
\boldsymbol{K}_{n 2}=\boldsymbol{K}_{22}+\boldsymbol{K}_{12}^{\mathrm{T}}\left[\left[\begin{array}{cc}
\boldsymbol{K}_{U} & \mathbf{0} \\
\mathbf{0} & \mathbf{0}
\end{array}\right]-\boldsymbol{K}_{22}\right]^{-1} \boldsymbol{K}_{12}
$$

where $\boldsymbol{K}_{12}$ and $\boldsymbol{K}_{22}$ are the $4 \times 4$ sub-matrices of $\overline{\boldsymbol{K}}_{e}, \overline{\boldsymbol{K}}_{e}=\left[\begin{array}{ll}\boldsymbol{K}_{11} & \boldsymbol{K}_{12} \\ \boldsymbol{K}_{12}^{\mathrm{T}} & \boldsymbol{K}_{22}\end{array}\right]$.

Based upon the aforementioned analysis, the position and pose of the end point (node 2) of UP limb must satisfy the deformation compatibility condition, and then the following equation can be formulated according to Eq. (19).

$$
{ }^{1} \mathbf{\$}_{t b}=\boldsymbol{T}_{b}\left(\begin{array}{c}
\boldsymbol{\Delta}_{p 2} \\
\boldsymbol{\Delta}_{\alpha 2}
\end{array}\right)
$$

where $\boldsymbol{\Delta}_{p 2}$ and $\boldsymbol{\Delta}_{\alpha 2}$ denote the linear and angular deformation of node 2 , and

$$
\boldsymbol{T}_{b}=\left[\begin{array}{c}
\boldsymbol{E}_{2} \\
-\boldsymbol{T}_{\alpha}^{+} \boldsymbol{T}_{p}
\end{array}\right], \boldsymbol{T}_{p}=\left[\begin{array}{c}
\left(t_{2} \boldsymbol{w}_{1}-t_{1} \boldsymbol{w}_{2}\right)^{\mathrm{T}} \\
\left(t_{4} \boldsymbol{w}_{3}-t_{3} \boldsymbol{w}_{4}\right)^{\mathrm{T}} \\
\boldsymbol{w}_{5}^{\mathrm{T}}
\end{array}\right]\left[\begin{array}{ll}
\boldsymbol{u} & \boldsymbol{v}
\end{array}\right], \quad \boldsymbol{T}_{\alpha}=\left[\begin{array}{c}
\left(\boldsymbol{a}_{1} \times t_{2} \boldsymbol{w}_{1}-\boldsymbol{a}_{2} \times t_{1} \boldsymbol{w}_{2}\right)^{\mathrm{T}} \\
\left(\boldsymbol{a}_{3} \times t_{4} \boldsymbol{w}_{3}-\boldsymbol{a}_{4} \times t_{3} \boldsymbol{w}_{4}\right)^{\mathrm{T}} \\
\left(\boldsymbol{a}_{5} \times \boldsymbol{w}_{5}\right)^{\mathrm{T}}
\end{array}\right]\left[\begin{array}{ll}
\boldsymbol{u} & \boldsymbol{v}
\end{array}\right]
$$

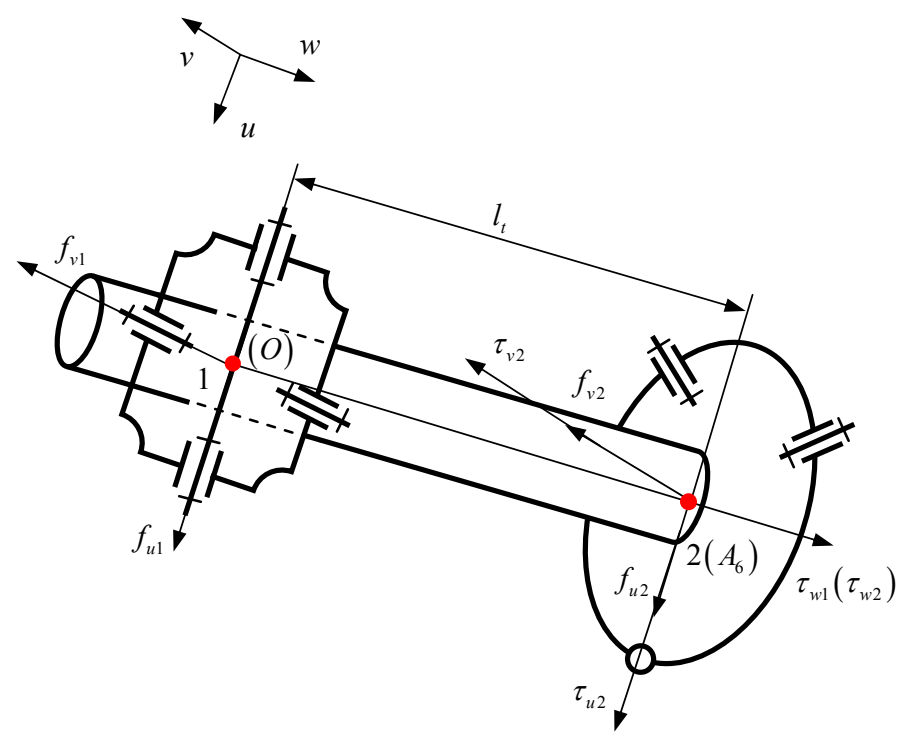

Fig. 6 Free-body diagram of UP limb

Bearing virtual work principle and Hooke's law in mind, the following equations are formulated 


$$
\left(\begin{array}{l}
\boldsymbol{\Delta}_{p 2} \\
\boldsymbol{\Delta}_{\alpha 2}
\end{array}\right)^{\mathrm{T}}\left(\begin{array}{l}
\boldsymbol{f}_{2} \\
\boldsymbol{\tau}_{2}
\end{array}\right)={ }^{1} \mathbf{S}_{t b}^{\mathrm{T}}{ }^{1} \mathbf{S}_{w b}
$$

where

$$
\left(\begin{array}{l}
\boldsymbol{f}_{2} \\
\boldsymbol{\tau}_{2}
\end{array}\right)=\boldsymbol{K}_{n 2}\left(\begin{array}{c}
\boldsymbol{\Delta}_{p 2} \\
\boldsymbol{\Delta}_{\alpha 2}
\end{array}\right),{ }^{1} \mathbf{S}_{t b}={ }^{1} \boldsymbol{C}_{b}{ }^{1} \mathbf{S}_{w b}
$$

herein, $\boldsymbol{f}_{2}$ and $\boldsymbol{\tau}_{2}$ denote the shearing force and the bending moment applied on node 2, ${ }^{1} \boldsymbol{\$}_{w b}$ represents the shearing force and the bending moment of UP limb.

Substituting Eq. (24) (25) into Eq. (26), leads to

$$
{ }^{1} \boldsymbol{C}_{b}=\boldsymbol{B} \boldsymbol{T}_{b}\left(\boldsymbol{T}_{b}^{\mathrm{T}} \boldsymbol{K}_{n 2} \boldsymbol{T}_{b}\right)^{-1} \boldsymbol{T}_{b}^{\mathrm{T}} \boldsymbol{B}^{\mathrm{T}}
$$

where $B=\left[\begin{array}{cc}{\left[\begin{array}{cc}u & v\end{array}\right]} & \mathbf{0}_{3 \times 2} \\ \mathbf{0}_{3 \times 2} & {\left[\begin{array}{ll}\boldsymbol{u} & \boldsymbol{v}\end{array}\right]}\end{array}\right]$.

The torsional deformation of UP limb can be obtained by the superposition of the angular deformation of $\mathrm{U}$ joint about $w$-axis and the torsional deformation of $\mathrm{P}$ joint. It is easy to derive

$$
{ }^{1} \mathbf{S}_{t t}={ }^{1} \boldsymbol{C}_{t}{ }^{1} \mathbf{S}_{w t}
$$

where

$$
{ }^{1} \boldsymbol{C}_{t}=\boldsymbol{T}_{t}^{1} \boldsymbol{C}_{w t} \boldsymbol{T}_{t}^{\mathrm{T}}, \boldsymbol{T}_{t}=\left[\begin{array}{cc}
\boldsymbol{R} & \mathbf{0} \\
\mathbf{0} & \boldsymbol{R}
\end{array}\right],{ }^{1} \boldsymbol{C}_{w t}=\operatorname{diag}\left(0,0,0,0,0, c_{t 1}+c_{t 2}\right), c_{t 1}=k_{\alpha w, \text { in }}^{-1}+\left(\boldsymbol{w}_{\text {out }}^{\mathrm{T}} \boldsymbol{K}_{\alpha, \text { out }} \boldsymbol{w}_{\text {out }}\right)^{-1}, c_{t 1}=\frac{l_{t}}{G I_{t}}
$$

herein, $k_{\alpha w, i n}$ denotes the angular stiffness of the proximal axis of $\mathrm{U}$ joint about $w$-axis, $\boldsymbol{K}_{\alpha, \text { out }}$ represents the angular stiffness matrix of the distal axis of $\mathrm{U}$ joint, $\boldsymbol{w}_{\text {out }}$ is the third column of orientation matrix of distal axis component with respect to proximal axis component, $l_{t}$ denotes the torsional length as shown in Fig. 6, and $G I_{t}$ represents the torsional section modulus of the beam element.

\subsection{Substructure II}

As stated in Section 2, the substructure II is a 2-DoF overconstrained parallel mechanism from the viewpoint of topology structure, whose compliance matrix can be considered as that formulates the compliance matrices of two closed-loops in their each reference frame and then obtains the compliance matrix of substructure II by means of the virtual work principle and Jacobian matrix.

It is obvious from Fig. 7 that the closed-loop I is exerted by three forces along $\bar{u}_{1}, \bar{v}_{1}$ and $\bar{w}_{1}$ axis and two moments about $\bar{v}_{1}$ and $\bar{w}_{1}$ axis in the local frame $D-\bar{u}_{1} \bar{v}_{1} \bar{w}_{1}$. As shown in Fig. 7, the parallelogram $E_{1} E_{2} E_{3} E_{4}$ can demonstrate the deformation transmission distinctly from $\mathrm{IR}_{1}$ to Point $D$ based upon the mechanical principle. In other words, the deformation of point $A_{6}$ can be transferred to point $D$ in two roads simultaneously in terms of road $A_{6} E_{1} E_{4} D$ and road $A_{6} E_{2} E_{3} D$. In consequence, the compliance matrix of closed-loop I considering the deformation of $\mathrm{IR}_{1}$ in frame $D-\bar{u}_{1} \bar{v}_{1} \bar{w}_{1}$ can be formulated as 


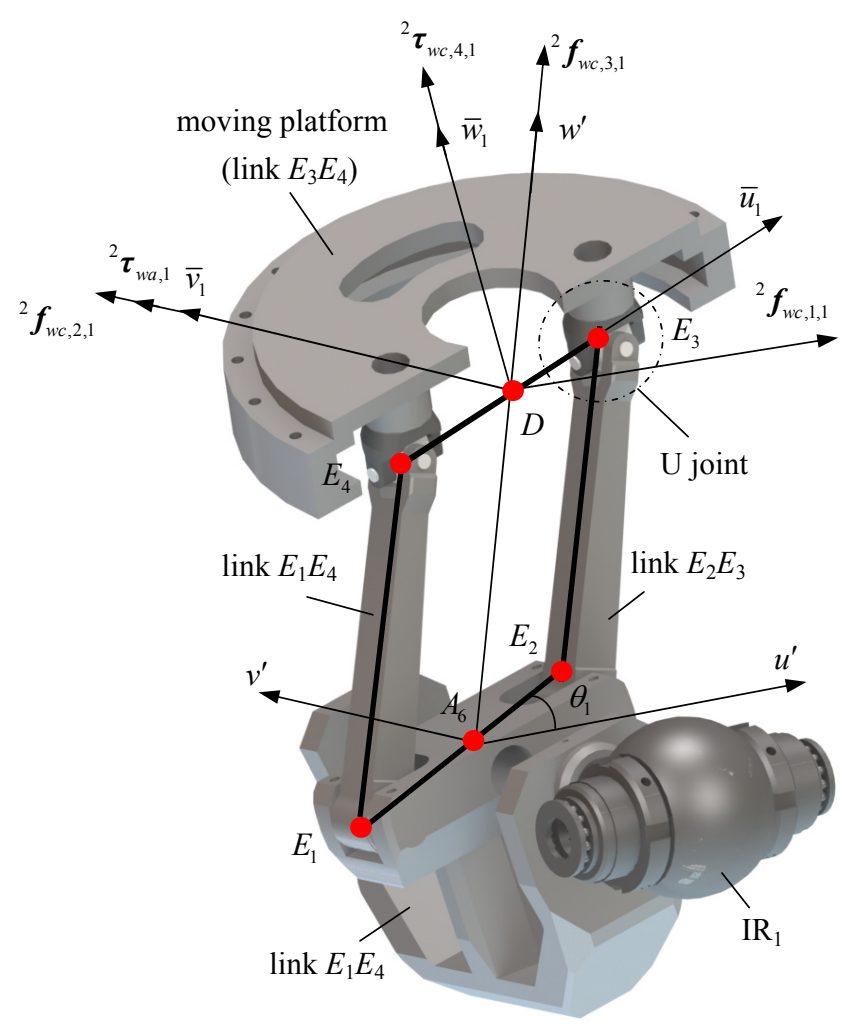

Fig. 7 3-D model of close-loop I

$$
\left\{\begin{array}{l}
{ }^{2} \boldsymbol{C}_{C L, 1,1}={ }^{2} \overline{\boldsymbol{C}}_{a c, 1}+{ }^{2} \overline{\boldsymbol{C}}_{1,1}+{ }^{2} \overline{\boldsymbol{C}}_{2,1}+{ }^{2} \overline{\boldsymbol{C}}_{3,1}+{ }^{2} \overline{\boldsymbol{C}}_{5,1} \\
{ }^{2} \boldsymbol{C}_{C L, 2,1}={ }^{2} \overline{\boldsymbol{C}}_{a c, 1}+{ }^{2} \overline{\boldsymbol{C}}_{1,1}+{ }^{2} \overline{\boldsymbol{C}}_{2,1}+{ }^{2} \overline{\boldsymbol{C}}_{4,1}+{ }^{2} \overline{\boldsymbol{C}}_{5,1}
\end{array}\right.
$$

where ${ }^{2} \overline{\boldsymbol{C}}_{1,1}$ denotes the compliance matrix of link $E_{1} E_{4}$ from IR 1 to point $A_{6},{ }^{2} \overline{\boldsymbol{C}}_{2,1}$ is the compliance matrix of link $E_{1} E_{4}$ from point $A_{6}$ to point $E_{1}$ or point $E_{2},{ }^{2} \overline{\boldsymbol{C}}_{3,1}$ and ${ }^{2} \overline{\boldsymbol{C}}_{4,1}$ represent the compliance matrices of link $E_{1} E_{4}$ and $E_{2} E_{3}$, respectively, ${ }^{2} \overline{\boldsymbol{C}}_{5,1}$ represents the compliance matrix of $\mathrm{U}$ joint of closed-loop I.

The virtual work equation of closed-loop I at point $D$ can be obtained as

$$
{ }^{2} \mathbf{S}_{t, C L 1}^{\mathrm{T}}{ }^{2} \mathbf{S}_{w, C L 1}={ }^{2} \mathbf{S}_{t, E_{3}}^{\mathrm{T}}{ }^{2} \boldsymbol{\$}_{w, E_{3}}+{ }^{2} \boldsymbol{\$}_{t, E_{4}}^{\mathrm{T}}{ }^{2} \boldsymbol{\$}_{w, E_{4}}
$$

Based upon the Hooke's law,

$$
{ }^{2} \boldsymbol{C}_{C L, j, 1}{ }^{2} \mathbf{\$}_{w, E_{i}}={ }^{2} \boldsymbol{\$}_{t, E_{i}}
$$

where ${ }^{2} \boldsymbol{\$}_{t, D}=\boldsymbol{T}_{E_{i}}{ }^{2} \boldsymbol{S}_{t, E_{i}}$ is the deformation compatibility conditions of point $E_{3}$ and $E_{4}, \boldsymbol{T}_{E_{i}}=\left[\begin{array}{cc}\boldsymbol{E}_{3} & -\left[\boldsymbol{l}_{E_{i}} \times\right] \\ \mathbf{0} & \boldsymbol{E}_{3}\end{array}\right], \boldsymbol{l}_{E_{i}}$ denotes the vector from point $E_{i}$ to point $D$ in frame $D-\bar{u}_{1} \bar{v}_{1} \bar{w}_{1} \cdot(j=1$ for $i=3 ; j=2$ for $i=4)$

Substituting Eq. (31) into Eq. (30), the stiffness matrix of closed-loop I in frame $D-\bar{u}_{1} \bar{v}_{1} \bar{w}_{1}$ can be formulated as 


$$
{ }^{2} \boldsymbol{K}_{C L, 1}=\sum_{j=1}^{2} \boldsymbol{T}_{E_{j}}^{-\mathrm{T} 2} \boldsymbol{C}_{C L, j, 1}^{-1} \boldsymbol{T}_{E_{j}}^{-1}
$$

As shown in Fig. 8, the closed-loop II is exerted by three forces along $\bar{u}_{2}, \bar{v}_{2}$ and $\bar{w}_{2}$ axis and one moment about $\bar{u}_{1}$ axis in the local frame $D-\bar{u}_{2} \bar{v}_{2} \bar{w}_{2}$. Similar to closed-loop I, the deformation of point $A_{6}$ can be transferred to point $D$ in road $A_{6} F_{1} F_{4} D$ and $\operatorname{road} A_{6} F_{2} F_{3} D$ simultaneously. The compliance matrix of closed-loop II considering $\mathrm{IR}_{2}$ in frame $D-\bar{u}_{2} \bar{v}_{2} \bar{w}_{2}$ can be calculated as

$$
\left\{\begin{array}{l}
{ }^{2} \overline{\boldsymbol{C}}_{C L, 1,2}={ }^{2} \overline{\boldsymbol{C}}_{a c, 2}+{ }^{2} \overline{\boldsymbol{C}}_{1,2}+{ }^{2} \overline{\boldsymbol{C}}_{2,2}+{ }^{2} \overline{\boldsymbol{C}}_{3,2}+{ }^{2} \overline{\boldsymbol{C}}_{5,2} \\
{ }^{2} \overline{\boldsymbol{C}}_{C L, 2,2}={ }^{2} \overline{\boldsymbol{C}}_{a c, 2}+{ }^{2} \overline{\boldsymbol{C}}_{1,2}+{ }^{2} \overline{\boldsymbol{C}}_{2,2}+{ }^{2} \overline{\boldsymbol{C}}_{4,2}+{ }^{2} \overline{\boldsymbol{C}}_{5,2}
\end{array}\right.
$$

where ${ }^{2} \overline{\boldsymbol{C}}_{1,2}$ denotes the compliance matrix of link $F_{1} F_{4}$ from IR 2 to point $A_{6},{ }^{2} \overline{\boldsymbol{C}}_{2,2}$ is the compliance matrix of link $F_{1} F_{4}$ from $A_{6}$ to point $F_{1}$ or point $F_{2},{ }^{2} \overline{\boldsymbol{C}}_{3,2}$ and ${ }^{2} \overline{\boldsymbol{C}}_{4,2}$ represent the compliance matrix of link $F_{1} F_{4}$ and $F_{2} F_{3},{ }^{2} \overline{\boldsymbol{C}}_{5,2}$ represents the compliance matrix of U joint of closed-loop II.

Bearing virtual work principle in mind, the following equation can be obtained as

$$
{ }^{2} \mathbf{S}_{t, C L 2}^{\mathrm{T}}{ }^{2} \boldsymbol{S}_{w, C L 2}={ }^{2} \boldsymbol{S}_{t, F_{3}}^{\mathrm{T}}{ }^{2} \boldsymbol{S}_{w, F_{3}}+{ }^{2} \boldsymbol{S}_{t, F_{4}}^{\mathrm{T}}{ }^{2} \boldsymbol{S}_{w, F_{4}}
$$

Based upon the Hooke's law,

$$
{ }^{2} \overline{\boldsymbol{C}}_{C L, j, 2}{ }^{2} \mathbf{\$}_{w, F_{i}}={ }^{2} \mathbf{S}_{t, F_{i}}
$$

where $j=1$ for $i=3, j=2$ for $i=4$.

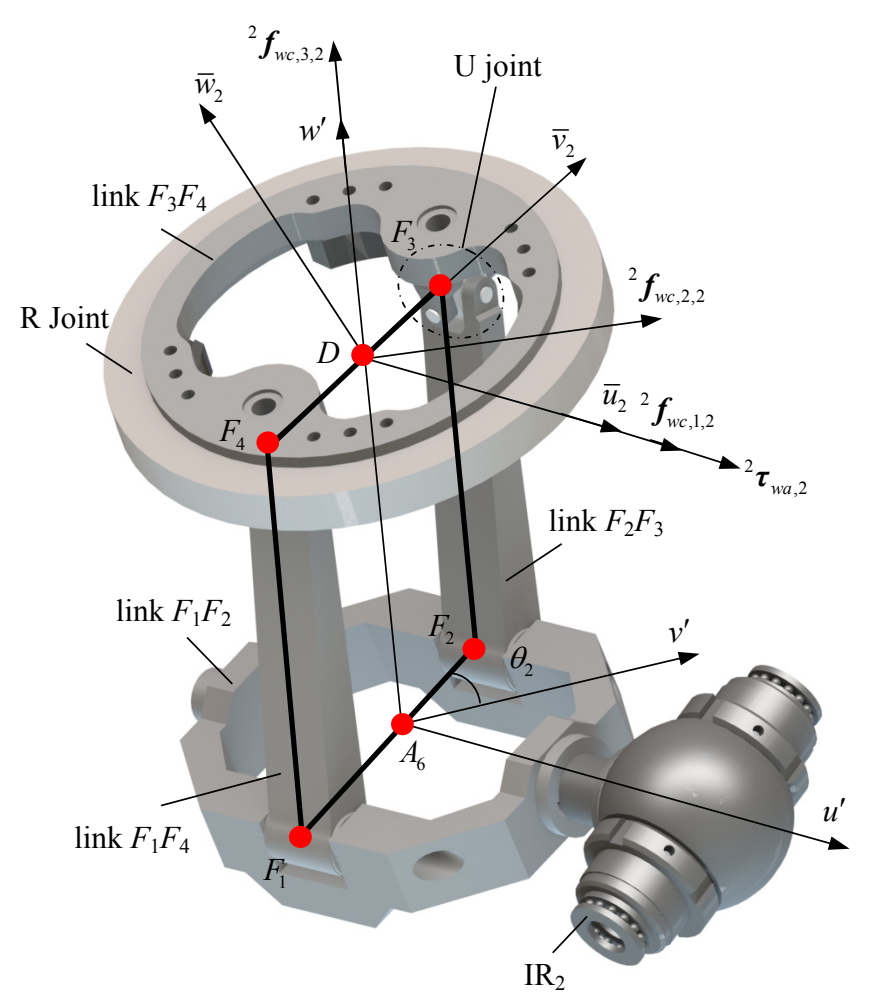

Fig. 8 3-D model of close-loop II 
Noting that the close-loop I and II is articulated by one R joint, the deformation compatibility conditions of closed-loop II at point $D$ can be given as

$$
{ }^{2} \boldsymbol{\$}_{t, D}=\boldsymbol{T}_{C L, 2} \boldsymbol{T}_{F_{i}}{ }^{2} \boldsymbol{\$}_{t, F_{i}}
$$

where $\boldsymbol{T}_{F_{i}}=\left[\begin{array}{cc}\boldsymbol{E}_{3} & -\left[\boldsymbol{l}_{F_{i}} \times\right] \\ \mathbf{0} & \boldsymbol{E}_{3}\end{array}\right], \boldsymbol{T}_{C L, 2}=\left[\begin{array}{cc}\boldsymbol{E}_{5} & \mathbf{0}_{5 \times 1} \\ \mathbf{0}_{1 \times 5} & 0\end{array}\right], \boldsymbol{l}_{F_{i}}$ denotes the vector from point $F_{i}$ to point $D$ in frame $D-\bar{u}_{2} \bar{v}_{2} \bar{w}_{2}, \boldsymbol{E}_{5}$ is $5 \times 5$ unit matrix.

Substituting Eq. (35) (36) into Eq. (34), the stiffness matrix of closed-loop II in frame $D-\bar{u}_{2} \bar{v}_{2} \bar{w}_{2}$ without link $F_{3} F_{4}$ can be formulated as

$$
{ }^{2} \overline{\boldsymbol{K}}_{C L, 2}=\sum_{j=1}^{2} \boldsymbol{T}_{C L, 2}^{-\mathrm{T}} \boldsymbol{T}_{F_{i}}^{-\mathrm{T} 2} \boldsymbol{C}_{C L, j, 2}^{-1} \boldsymbol{T}_{F_{i}}^{-1} \boldsymbol{T}_{C L, 2}^{-1}
$$

Taking compliance matrix ${ }^{2} \overline{\boldsymbol{C}}_{F_{3} F_{4}}$ of link $F_{3} F_{4}$ into account, the stiffness matrix of closed-loop II at point $D$ is obtained by linear superposition principle.

$$
{ }^{2} \boldsymbol{K}_{C L, 2}=\left({ }^{2} \overline{\boldsymbol{K}}_{C L, 2}^{-1}+{ }^{2} \overline{\boldsymbol{C}}_{F_{3} F_{4}}\right)^{-1}
$$

The virtual work equation of substructure II at point $D$ considering deformations of closed-loop I and II simultaneously can be obtained as

$$
{ }^{2} \boldsymbol{S}_{t, D}^{\mathrm{T}}{ }^{2} \boldsymbol{S}_{w, D}={ }^{2} \boldsymbol{S}_{t, C L, 1}^{\mathrm{T}}{ }^{2} \boldsymbol{\$}_{w, C L, 1}+{ }^{2} \mathbf{S}_{t, C L, 2}^{\mathrm{T}}{ }^{2} \mathbf{S}_{w, C L, 2}
$$

On the basis of Jacobian matrices of closed-loop I, II and Hooke's law, the compliance matrix of substructure II at point $D$ without moving platform in frame $D-u^{\prime} v^{\prime} w^{\prime}$ can be described by

$$
{ }^{2} \overline{\boldsymbol{C}}=\left(\sum_{i=1}^{2} \boldsymbol{J}_{C L, i}^{\mathrm{T}}{ }^{2} \boldsymbol{K}_{C L, i} \boldsymbol{J}_{C L, i}\right)^{-1}
$$

where

$$
\boldsymbol{J}_{C L, 1}=\left[\begin{array}{cc}
{ }^{2} \boldsymbol{s}_{1,2}^{\mathrm{T}} & \mathbf{0} \\
{ }^{2} \boldsymbol{s}_{1,1}^{\mathrm{T}} & \mathbf{0} \\
\left({ }^{2} \boldsymbol{s}_{1,2} \times{ }^{2} \boldsymbol{s}_{1,1}\right)^{\mathrm{T}} & \mathbf{0} \\
\mathbf{0} & { }^{2} \boldsymbol{s}_{1,1}^{\mathrm{T}} \\
\mathbf{0} & \left({ }^{2} \boldsymbol{s}_{1,2} \times{ }^{2} \boldsymbol{s}_{1,1}\right)^{\mathrm{T}}
\end{array}\right], \boldsymbol{J}_{C L, 2}=\left[\begin{array}{ccc}
{ }^{2} \boldsymbol{s}_{1,2}^{\mathrm{T}} /\left({ }^{2} \boldsymbol{s}_{2,2} \times{ }^{2} \boldsymbol{s}_{3,2}\right) & \mathbf{0} \\
{ }^{2} \boldsymbol{s}_{1,1}^{\mathrm{T}} /\left({ }^{2} \boldsymbol{s}_{2,2} \times{ }^{2} \boldsymbol{s}_{3,2}\right) & \mathbf{0} \\
\left({ }^{2} \boldsymbol{s}_{1,2} \times{ }^{2} \boldsymbol{s}_{1,1}\right)^{\mathrm{T}} /\left({ }^{2} \boldsymbol{s}_{2,2} \times{ }^{2} \boldsymbol{s}_{3,2}\right) & \mathbf{0} \\
\mathbf{0} & { }^{2} \boldsymbol{s}_{1,2}^{\mathrm{T}} /\left({ }^{2} \boldsymbol{s}_{2,2} \times{ }^{2} \boldsymbol{s}_{3,2}\right)
\end{array}\right]
$$

The compliance matrix of moving platform ${ }^{2} \overline{\boldsymbol{C}}_{E_{3} E_{4}}$ is considered by linear superposition principle, and then the complia nce matrix of substructure II is derived as

$$
{ }^{2} \boldsymbol{C}={ }^{2} \overline{\boldsymbol{C}}+\boldsymbol{T}_{R \theta}{ }^{2} \overline{\boldsymbol{C}}_{E_{3} E_{4}} \boldsymbol{T}_{R \theta}^{\mathrm{T}}
$$


where $\boldsymbol{T}_{R \theta}=\left[\begin{array}{cc}\boldsymbol{R}_{\theta} & \mathbf{0} \\ \mathbf{0} & \boldsymbol{R}_{\theta}\end{array}\right]$.

\section{Stiffness experiment}

In order to verify the stiffness model of T5 PKM considering gravitational effects, stiffness experiment is carried out in this section. Based upon the kinematic optical design of T5 PKM in [19], its dimensional parameters and prescribed workspace are given in Table 1. The inertial parameters are obtained and hereby the virtual prototype of T5 PKM is established by commercial software. On the basis of the virtual prototype of T5 PKM, the compliance coefficients, mass and mass centers of required components in the Section 3 and 4 are measured shown in Table 2 8.

Table 1 Dimensional parameters and workspace of T5 PKM

\begin{tabular}{ccccccccc}
\hline \multicolumn{4}{c}{ Dimensional parameters $(\mathrm{mm})$} & \multicolumn{6}{c}{ workspace } \\
\hline$b$ & $a$ & $b_{0}$ & $h_{0}$ & $d$ & $H(\mathrm{~mm})$ & $R(\mathrm{~mm})$ & $h(\mathrm{~mm})$ & $\varphi_{\max }(\circ)^{\mathrm{a}}$ \\
400 & 167 & 136 & 111 & 167.5 & 1200 & 400 & 300 & 40 \\
\hline${ }^{\mathrm{a}} \varphi_{\max }$ is the maximum rotational angle of $\mathrm{T} 5$ & PKM about $x$ or $y$-axis
\end{tabular}

Table 2 Compliance coefficients of UPSS limbs (unit: $\left.(\mu \mathrm{m} / \mathrm{N}) \times 10^{-3}\right)$

\begin{tabular}{lccccccccc}
\hline$c_{s, 1}$ & $c_{s, 2}$ & $c_{s, 3, g r}{ }^{\mathrm{a}}$ & $c_{s, 3, q 5}{ }^{\mathrm{b}}$ & $c_{a, i, 2}$ & $c_{a, i, 3}$ & $c_{a, i, 5}$ & $c_{a, i, 6}$ & $c_{h, \text { in }}$ & $c_{h, \text { out }}$ \\
\hline 37.520 & 90.907 & 12.518 & 25.980 & 12.831 & 10.571 & 2.500 & 3.770 & 5.072 & 4.055 \\
\hline${ }^{\mathrm{a}} c_{s, 3, g r}$ & is linear compliance of part 3 of S joints from $j$ th UPS limb $(j=1,2, \cdots, 4)$ \\
${ }^{\mathrm{b}} c_{s, 3, q 5}$ is linear compliance of part 3 of S joints from 5th UPS limb
\end{tabular}

Table 3 Lead screw parameters and compliance of R joints

\begin{tabular}{|c|c|c|c|c|c|c|c|}
\hline \multicolumn{2}{|c|}{ Lead screw Parameters } & \multicolumn{3}{|c|}{$\begin{array}{l}\text { Linear Compliance } \\
(\mu \mathrm{m} / \mathrm{N}) \times 10^{-3}\end{array}$} & \multicolumn{3}{|c|}{$\begin{array}{l}\text { Angular Compliance } \\
(\mathrm{rad} /(\mathrm{N} \cdot \mathrm{m})) \times 10^{-6}\end{array}$} \\
\hline$L_{s c}(\mathrm{~mm})$ & $E A_{s c}(\mathrm{MN})$ & $u^{\prime}$ & $v^{\prime}$ & $w^{\prime}$ & $u^{\prime}$ & $v^{\prime}$ & $w^{\prime}$ \\
\hline 688 & 44.12 & 86.03 & 299.7 & 43.01 & 12.89 & $\infty$ & 16.52 \\
\hline
\end{tabular}

Table 4 Compliance coefficients of UP limb

\begin{tabular}{cccccccc}
\hline$c_{u, \text { in }}$ & $c_{v, \text { in }}$ & $c_{w, \text { in }}$ & $c_{u, \text { out }}$ & $c_{v, \text { out }}$ & $c_{w, \text { out }}$ & $E I(\mathrm{MN})$ & $G_{t}(\mathrm{MN})$ \\
\hline 0.787 & 34.843 & 1.190 & 4.310 & 4.762 & 0.041 & 2.852 & 1.103 \\
\hline
\end{tabular}

Table 5 Component compliance coefficients of closed-loop I in substructure II

\begin{tabular}{ccccccc}
\hline & \multicolumn{2}{c}{ Linear Compliance $(\mu \mathrm{m} / \mathrm{N}) \times 10^{-3}$} & \multicolumn{4}{c}{ Angular Compliance $(\mathrm{rad} /(\mathrm{N} \cdot \mathrm{m})) \times 10^{-6}$} \\
& $\bar{u}_{1}$ & $\bar{v}_{1}$ & $\bar{w}_{1}$ & $\bar{u}_{1}$ & $\bar{v}_{1}$ & $\bar{w}_{1}$ \\
\hline${ }^{2} \overline{\boldsymbol{C}}_{a c, 1}$ & 1.076 & 0.357 & 1.213 & 0.231 & $\infty$ & 0.0117 \\
${ }^{2} \overline{\boldsymbol{C}}_{1,1}$ & 48.408 & 965.38 & 0.102 & 0.176 & $\infty$ & 0.0102 \\
${ }^{2} \overline{\boldsymbol{C}}_{2,1}$ & 0.465 & 1.938 & 2.009 & $\infty$ & $\infty$ & 0.0150 \\
${ }^{2} \overline{\boldsymbol{C}}_{3,1}\left({ }^{2} \overline{\boldsymbol{C}}_{4,1}\right)$ & $\infty$ & 97.176 & 1.531 & $\infty$ & $\infty$ & 0.00172 \\
\hline
\end{tabular}




\begin{tabular}{ccccccc}
\hline${ }^{2} \overline{\boldsymbol{C}}_{5,1}$ & 0.0612 & 0.0642 & 0.1184 & $\infty$ & $\infty$ & 0.00473 \\
${ }^{2} \overline{\boldsymbol{C}}_{E_{3} E_{4}}$ & 23.634 & 87.346 & 3.213 & 0.0274 & $\infty$ & 0.316 \\
\hline
\end{tabular}

Table 6 Component compliance coefficients of closed-loop II in substructure II

\begin{tabular}{ccccccc}
\hline & \multicolumn{2}{c}{ Linear Compliance $(\mu \mathrm{m} / \mathrm{N}) \times 10^{-3}$} & \multicolumn{3}{c}{ Angular Compliance } \\
& $\bar{u}_{2}$ & $\bar{v}_{2}$ & $\bar{w}_{2}$ & $\bar{u}_{2}$ & $\bar{v}_{2}$ & $\bar{w}_{2}$ \\
\hline${ }^{2} \overline{\boldsymbol{C}}_{a c, 2}$ & 0.357 & 1.076 & 1.213 & $\infty$ & 0.231 & 0.0117 \\
${ }^{2} \overline{\boldsymbol{C}}_{1,2}\left({ }^{2} \overline{\boldsymbol{C}}_{2,2}\right)$ & 52.388 & $\infty$ & 18.834 & $\infty$ & 0.0612 & 0.0316 \\
${ }^{2} \overline{\boldsymbol{C}}_{3,2}\left({ }^{2} \overline{\boldsymbol{C}}_{4,2}\right)$ & 6.929 & $\infty$ & $\infty$ & $\infty$ & 0.0642 & 0.0917 \\
${ }^{2} \overline{\boldsymbol{C}}_{5,2}$ & 0.126 & $\infty$ & 0.104 & $\infty$ & 0.1184 & 0.0028 \\
${ }^{2} \overline{\boldsymbol{C}}_{F_{3} F_{4}}$ & 1.336 & 9.735 & 0.721 & 0.0132 & 0.0357 & 0.0142 \\
\hline
\end{tabular}

Table 7 Mass and center of mass of components in substructure I

\begin{tabular}{cccccccc}
\hline${ }^{1} m_{L_{1}}(\mathrm{~kg})$ & ${ }^{1} m_{L_{2}}(\mathrm{~kg})$ & ${ }^{1} m_{I R_{j a}}(\mathrm{~kg})$ & $\rho A_{C}(\mathrm{~kg} / \mathrm{m})$ & ${ }^{1} m_{P}(\mathrm{~kg})$ & ${ }^{1} l_{1}(\mathrm{~mm})$ & ${ }^{1} l_{2}(\mathrm{~mm})$ & ${ }^{1} l_{P}(\mathrm{~mm})$ \\
\hline 14.501 & 7.473 & 3.434 & 37.758 & 20.142 & 198.65 & 405.27 & 25.61 \\
\hline
\end{tabular}

Table 8 Mass and center of mass of components in substructure II

\begin{tabular}{ccccc}
\hline & \multirow{2}{*}{ Mass $(\mathrm{kg})$} & \multicolumn{3}{c}{ Coordinate of Central Point $(\mathrm{mm})$} \\
& & $\bar{u}_{i}$ & $\bar{v}_{i}$ & $\bar{w}_{i}$ \\
\hline $\operatorname{link} E_{1} E_{2}$ & 6.390 & 0.95 & -0.8 & -209.83 \\
$\operatorname{link} E_{1} E_{4}\left(E_{2} E_{3}\right)$ & 0.607 & $-60(60)$ & 0 & -96.46 \\
$\operatorname{link} E_{3} E_{4}$ & 3.582 & 0 & 0 & 26.95 \\
$\operatorname{link} F_{1} F_{2}$ & 2.839 & -4.07 & 0 & -173.89 \\
$\operatorname{link} F_{1} F_{4}\left(F_{2} F_{3}\right)$ & 0.861 & 0 & $65(-65)$ & -98.11 \\
$\operatorname{link} F_{3} F_{4}$ & 3.893 & 0 & 0 & 25.95 \\
$\mathrm{U}$ joint in closed-loop I & 0.133 & $-60(60)$ & 0 & 12.98 \\
$\mathrm{U}$ joint in closed-loop II & 0.133 & 0 & $65(-65)$ & 12.98 \\
\hline
\end{tabular}

Table 9 Configurations of 12 measuring points in frame $O-x y z$

\begin{tabular}{cccccc}
\hline & $x(\mathrm{~mm})$ & $y(\mathrm{~mm})$ & $z(\mathrm{~mm})$ & $\varphi_{x}(\circ)$ & $\varphi_{y}(\circ)$ \\
\hline$D_{1}$ & 0 & 0 & 1200 & 0 & 0 \\
$D_{2}$ & 0 & 0 & 1250 & 0 & 0 \\
$D_{3}$ & 0 & 0 & 1300 & 0 & 0 \\
$D_{4}(\theta=0)$ & 0 & -300 & 1200 & 0 & 0 \\
$D_{5}\left(\theta=45^{\circ}\right)$ & 212.132 & -212.132 & 1200 & 0 & 0 \\
$D_{6}\left(\theta=90^{\circ}\right)$ & 300 & 0 & 1200 & 0 & 0 \\
$D_{7}\left(\theta=135^{\circ}\right)$ & 212.132 & 212.132 & 1200 & 0 & 0 \\
$D_{8}\left(\theta=180^{\circ}\right)$ & 0 & 300 & 1200 & 0 & 0 \\
$D_{9}$ & 0 & 0 & 1200 & -40 & 0 \\
$D_{10}$ & 0 & 0 & 1200 & -20 & 0 \\
$D_{11}$ & 0 & 0 & 1200 & 20 & 0 \\
$D_{12}$ & 0 & 0 & 1200 & 40 & 0 \\
\hline
\end{tabular}




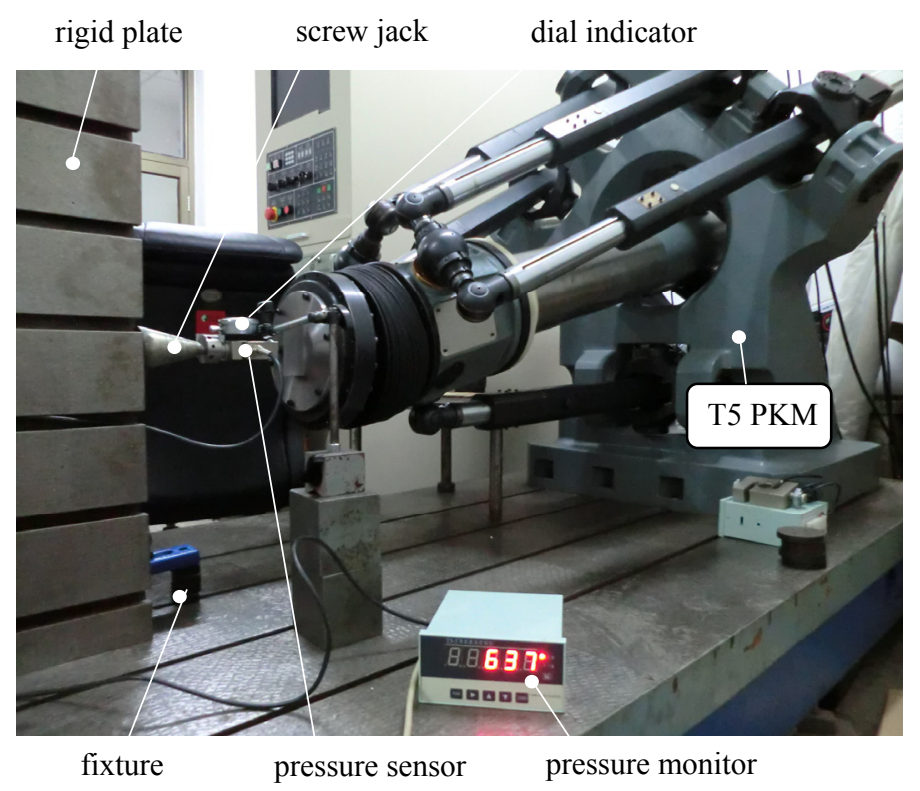

Fig. 9 Stiffness experiment of T5 PKM

Based upon the stiffness model of T5 PKM formulated in Section 3 and 4, the theoretical stiffness values of the reference point $D$ of T5 PKM in the prescribed workspace can be obtained easily utilizing the parameter values given in Table $1 \sim 8$.

To verify the theoretical stiffness model, the experimental stiffness values need to be measured by means of the stiffness experiment of T5 PKM. As shown in Fig. 9, the stiffness experiment flow of T5 PKM can be considered as that: 1) actuating the reference point $D$ of T5 PKM to a measuring point by means of five servomotors; 2) exerting external forces monitored by a pressure sensor to the reference point $D$ by means of the screw jack; 3) measuring the deformations of the reference point $D$ by means of the dial indicator; 4) obtaining the experimental stiffness values of the reference point $D$ by utilizing the division of the exerted forces and its corresponding deformations.




Fig. 10 Measuring points of stiffness experiment

Without loss of generality, the 12 measuring points of the reference point $D$ are selected, denoted by $D_{1} \sim D_{12}$ shown in Fig. 10 , and their configurations described in frame $O-x y z$ are shown in Table 9. It is pointed out that several tips in the stiffness experiment are carried out in order to decrease the measuring error: 1) exerting the preload for eliminating the effects of the clearances among components and joints $(200 \mathrm{~N}, 300 \mathrm{~N}$ and $400 \mathrm{~N}$ preloads are exerted along $x$-axis, $y$-axis and $z$-axis, respectively); 2) taking $50 \mathrm{~N}$ as the step length, the experimental stiffness curve is fitted by means of the least square method; 3) the stiffness value at any point along/about any axis is an average value after repeating four times.

The experimental stiffness values of the reference point at the 12 measuring points are measured and demonstrated in Fig. 11 with the comparison with the theoretical stiffness values, in which the red curve and blue curve denote the theoretical value and experimental value, respectively. The errors between the two models are within $5 \%$, and it is concluded that the variation tendency of the experimental values is similar to that of the theoretical values. The experimental values are smaller than the theoretical values in all configurations which may be caused by the friction and nonlinear contact deformation between two components in T5 PKM prototype.

In conclusion, the theoretical stiffness model of T5 PKM is proved as valid by means of the stiffness experimental approach. In other words, the stiffness modeling flow of T5 PKM is effective, and the approach that treating the component gravities as external payloads in the theoretical model can be employed to describe the actual distributed gravities in an acceptable accuracy manner. 

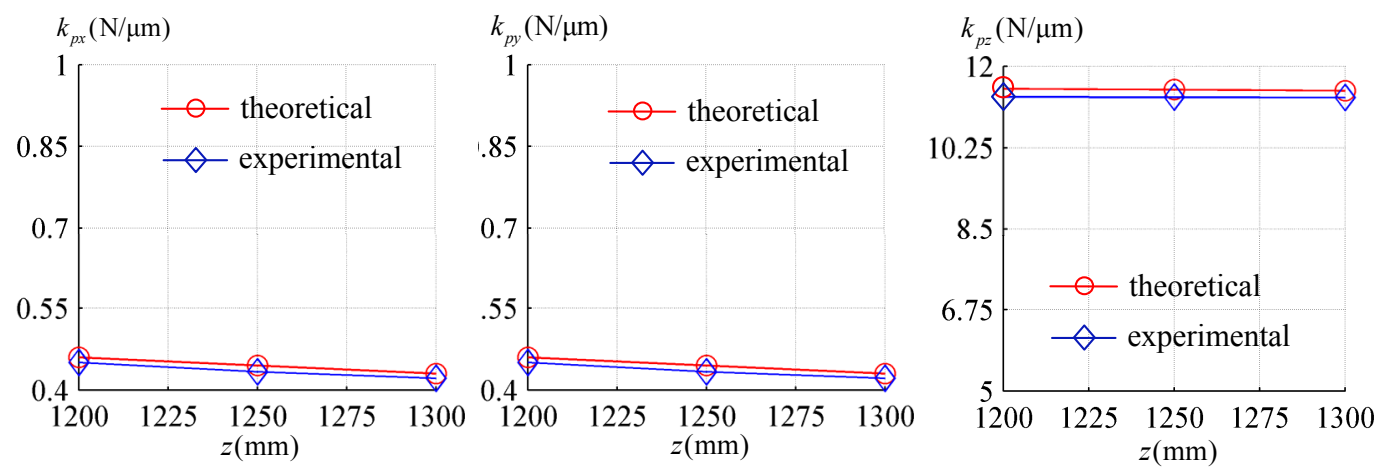

(a) Measuring points from $D_{1}$ to $D_{3}$
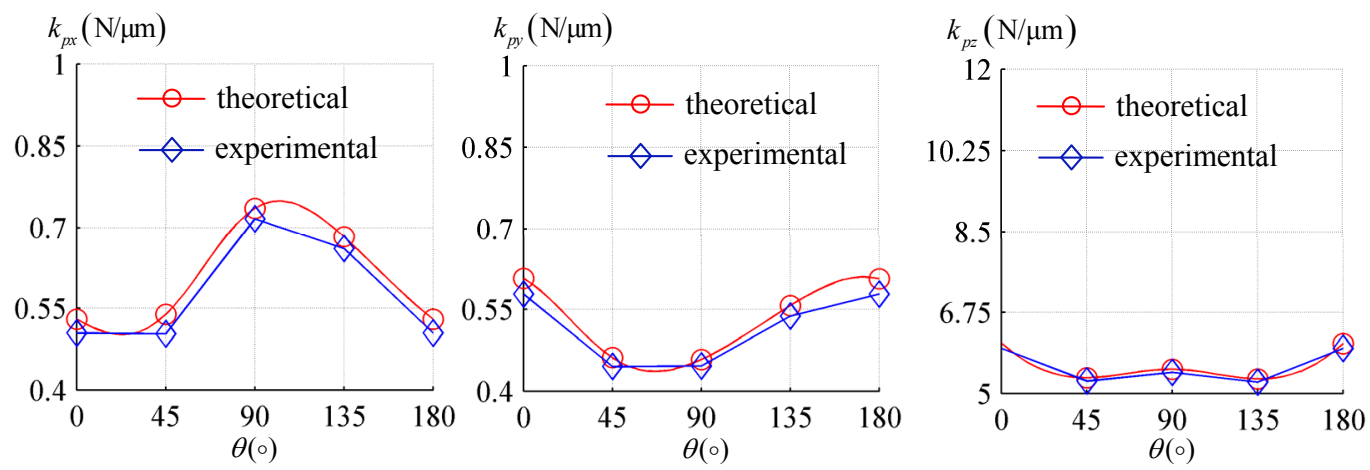

(b) Measuring points from $D_{4}$ to $D_{8}$
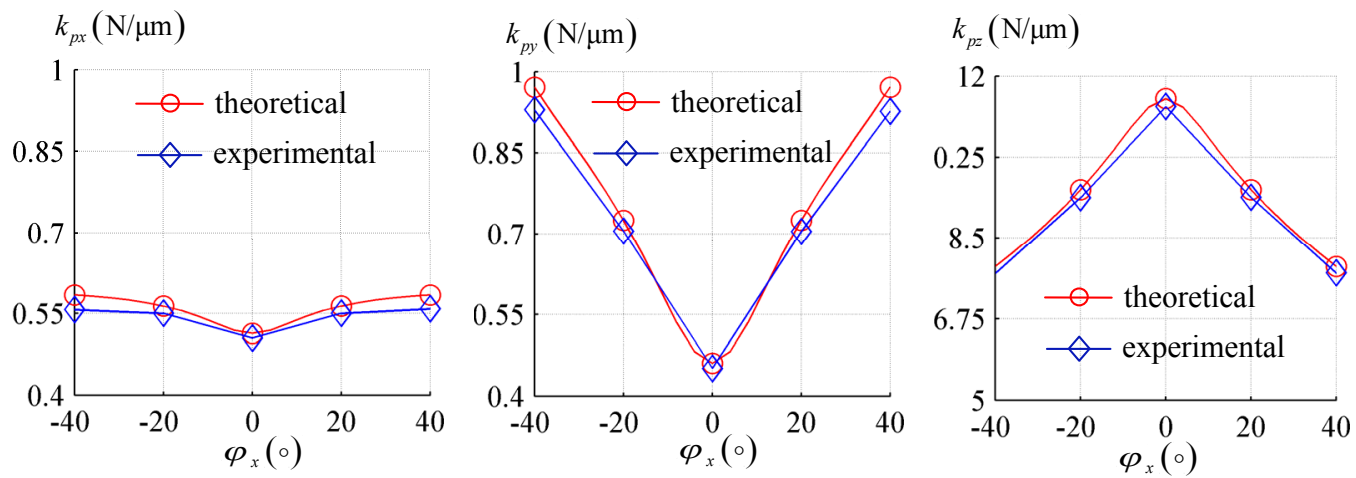

(c) Measuring points from $D_{9}$ to $D_{12}$

Fig 11 Stiffness comparison between theoretical and experimental stiffness values

\section{Stiffness analysis}

The stiffness distribution of T5 PKM in the prescribed workspace is presented as follow.

As shown in Fig. 12, the stiffness distribution of T5 PKM in three plane ( $z=1200, z=1350, z=1500 \mathrm{~mm})$ is presented when $\varphi_{x}=0$ and $\varphi_{y}=0$. It is easy and visual from Fig. 12 to find out that: 1) the linear and angular stiffness all decrease with increment of $z$ value; 2) the linear stiffness $k_{p x}, k_{p y}$ and $k_{p z}$ are distributed plane-symmetrically; 3) the distributions of $k_{p x}$ and $k_{p y}$ are symmetrical about plane $y=0$ and $x=0$, respectively, the values of $k_{p x}$ and $k_{p y}$ get their minimums near their symmetrical plane and obtain their maximums close to the workspace boundary; 4) the distribution of $k_{p z}$ is symmetrical about plane $y=0$, the 
value of $k_{p z}$ is much bigger than those of $k_{p x}$ and $\left.k_{p y} ; 5\right)$ the distributions of $k_{\alpha x}, k_{\alpha y}$ and $k_{\alpha z}$ are symmetrical about plane $y=-x ; 6)$ the variations of $k_{\alpha x}$ and $k_{\alpha y}$ are contrary to that of $k_{\alpha z}$.



(a)

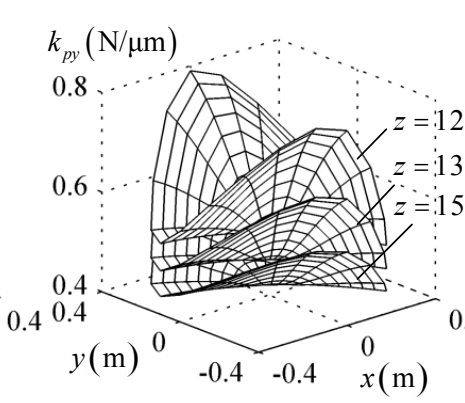

(b)

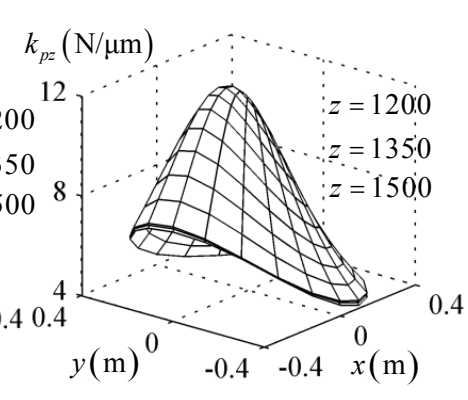

(c)

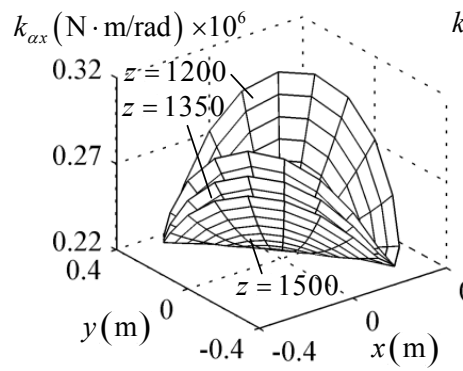

(d)

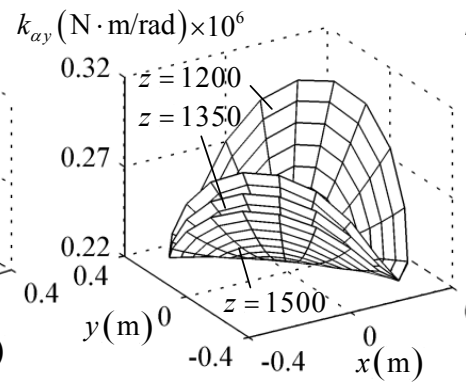

(e)

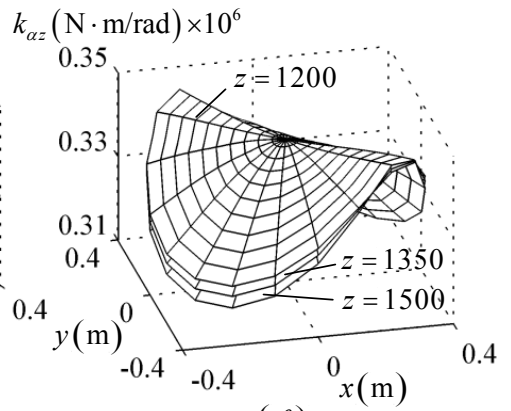

(f)

Fig. 12 Stiffness distribution in there planes $\left(\varphi_{x}=0, \varphi_{y}=0\right)$

It is noted that the stiffness model of T5 PKM is determined by compliances of $\mathrm{IR}_{1}$ and $\mathrm{IR}_{2}$, substructure I and II, hence these three parts should make appropriate stiffness contributions to the stiffness of T5 PKM in order to avoid bucket effect as mentioned above.

As shown in Fig. 13, the gravity of T5 PKM has a significant impact on the linear deformation $\Delta_{p x}$ along $x$-axis and the angular deformations $\Delta_{\alpha y}$ and $\Delta_{\alpha z}$ about $y$ and $z$-axis, therefore, the gravity of T5 PKM should not be ignored in the stiffness modeling. When concerning the gravitational contribution of substructure I and II, it is explicit that substructure I has greater effect to reference point $D$ since it accounts for larger proportion of gravity and is further away from reference point $D$. As shown in Fig. 14, the gravity contributions of UP limb and platform I, UPS limb to the deformation of reference point $D$ are demonstrated distinctly. In plane $z=1350$ of the prescribed workspace, $\Delta_{p x}, \Delta_{\alpha y}$ and $\Delta_{\alpha z}$ caused by UP limb is much bigger than the others, which means that it is essential to properly reduce the weight of UP limb without affecting the stiffness performance.

Therefore, the aforementioned stiffness modeling and analysis can lay a solid foundation for the parameterized stiffness design of T5 PKM in a separate research paper. 

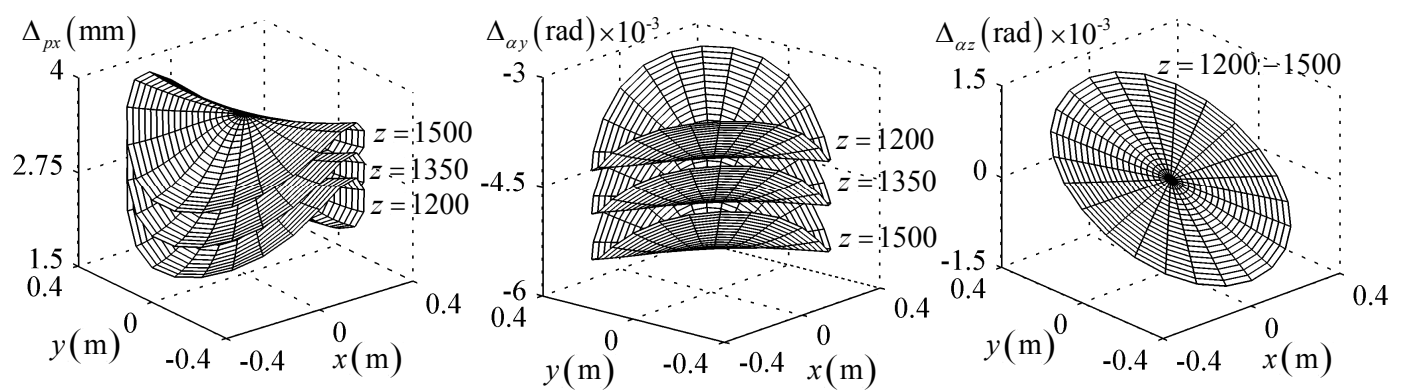

Fig 13 Deformations of reference point caused by gravity within prescribed workspace
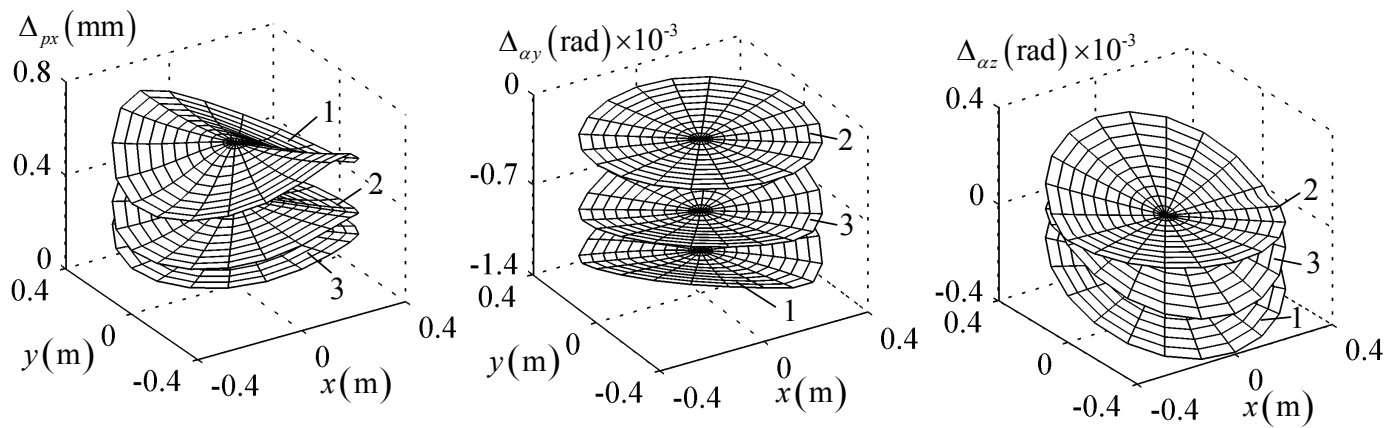

Fig 14 Deformations of reference point caused by gravity of 1- UP limb, 2- Platform I, 3-UPS limb I at $z=1350$

\section{Conclusion}

In order to carry out the high-precision machining of aerospace structural components with large size, thin wall and complex surface, this paper proposes a novel T5 PKM based solution and formulates the theoretical stiffness model of T5 PKM considering gravitational effects that is verified by experiments. The conclusions are drawn as follows.

(1) On the basis of 5-DoF PKMs exampled successfully by Tricept-like and Sprint Z3-like PKMs, this paper introduces a novel 5-DoF PKM whose servomotors are all arranged adjacent to the fixed base so as to reduce the weight of the 2-DoF rotating head and avoid integration with two long guide ways. The complete parallel structure makes it a promising solution for high precision machining.

(2) The semi-analytical stiffness modeling of T5 PKM is demonstrated in this paper, which firstly formulates the compliance models of Substructure I and II in their joint spaces, then maps these compliance models to the operated space by virtual work principle and Jacobian matrix, and finally obtains the stiffness model of T5 PKM based upon the deformation superposition principle. In the process, several common issues for other PKMs are solved including accurate stiffness modeling of R, $\mathrm{U}$ and $\mathrm{S}$ joints, reasonable stiffness extraction of components using FEA software and precise stiffness modeling of over-constrained mechanisms.

(3) The component gravities are treated as external payloads exerting on the end reference point by static equivalent principle during wrench analysis. The consideration of component gravities enables the semi-analytical stiffness model of T5 PKM to be more practical and reliable. The two interlinked $\mathrm{R}$ joints are the critical components so that their deformation contributions are taken into account to develop the compliance models of Substructure I and II, respectively.

(4) The stiffness experiment of T5 PKM is carried out to verify its theoretical stiffness model, and the reason why the experimental stiffness values are smaller than theoretical stiffness values is discussed. Thus the stiffness modeling in this paper would be referred by researchers when they deal with similar problems. 
By employing the theoretical stiffness model verified, the stiffness distributions of T5 PKM are illustrated and the contributions of component gravities to the stiffness of T5 PKM are discussed. These are used to analyze and evaluate the stiffness performance of T5 PKM. As the counterpart, the algebraic characteristics (such as singular values and eigenvalues) of the stiffness matrix are usually employed as the objective function or constraint conditions within the whole optimal design process. In addition, the analytical stiffness model in this paper would contribute to the digital design of PKMs by means of computer and the programming languages.

\section{Acknowledgments}

This research work was supported by the National Natural Science Foundation of China (NSFC) under Grant No. 51205278 and 51475321, Ph.D. Programs Foundation of Ministry of Education of China (Grant No. 2012003211003, 2012003212003), Tianjin Research Program of Application Foundation and Advanced Technology (Grant No. 13JCQNJC04600 and 15JCZDJC38900) and China Scholarship Council (CSC).

\section{Reference}

[1] M. Weck, D. Staimer, Parallel kinematic machine tool - current state and future potentials, CIRP Ann. - Manuf. Techn. 51 (2002) 671-683.

[2] M. Geldart, P. Webb, H. Larsson, M. Backstrom, N. Gindy, K. Rask, A direct comparison of the machining performance of a variax 5 axis parallel kinetic maching centre with conventional 3 and 5 axis machine tools, Int. J. Mach. Tools Manuf. 43 (2003) 1107-1116.

[3] M. Terrier, A. Dugas, J. Y. Hascoet, Qualification of parallel kinematics machines in high-speed milling on free form surfaces, Int. J. Mach. Tools Manuf. 44 (2004) 865-877.

[4] F. Gao, B.B. Peng, H. Zhao, W. M. Li, A novel 5-DoF parallel kinematic machine tool, Int. J. Mach. Tools Manuf. 31 (2006) 201-207.

[5] R. Katz, Z. Li, Kinematic and dynamic synthesis of a parallel kinematic high speed drilling machine, Int. J. Mach. Tools Manuf. 44 (2004) 1381-1389.

[6] P. L. Yen, A two-loop robust controller for compensation of the variant friction force in an over-constrained parallel kinematic machine, Int. J. Mach. Tools Manuf. 48 (2008) 1354-1365.

[7] T. Sun, Y. M. Song, G. Dong, B. B. Lian, J. P. Liu, Optimal design of a parallel mechanism with three rotational degrees of freedom, Robot. Com.-Int Manuf. 28 (4) (2012) 500-508.

[8] K.E. Neumann, System and method for controlling a robot, US Patent, No.6301525, 2001.

[9] F. Xi, D. Zhang, Z. Xu, C. M. Mechefske, A comparative study on tripod units for machine tools, Int. J. Mach. Tools Manuf. 43 (7) (2003) 721-730.

[10] H. Chanal, E. Duc, P. Ray, A study of the impact of machine tool structure on machining process, Int. J. Mach. Tools Manuf. 46 (2006) 98-106.

[11] W. Joachim, Articulated tool head, WO2000025976, 2000.

[12] X. Chen, X. J. Xiu, F. G. Xie, T. Sun, A comparison study on motion/force transmissibility of two typical 3-DoF parallel manipulators: the sprint Z3 and A3 tool heads, Int. J. Adv Robot. Syst 11 (5) (2014) DOI: 10. 5772/57458.

[13] B. Siciliano, The Tricept robot: inverse kinematics, manipulability analysis and closed-loop direct kinematics algorithm, Robotica 17 (1999) 437- 445 .

[14] T. Sun, Y. M. Song, Y. G. Li, J. Zhang, Workspace decomposition based dimensional synthesis of a novel hybrid reconfigurable robot, J. Mech. Robot. 2 (3) (2010) 031009-1-031009-8.

[15] Z. M. Bi, Y. Jin, Kinematic modeling of Exechon parallel kinematic machine, Robot. Com.-Int Manuf. 27 (2011) 186-193.

[16] T. Sun, Y. M. Song, Y. G. Li, L. S. Liu, Dimensional synthesis of a 3-DOF parallel manipulator based on dimensionally homogeneous Jacobian matrix, Sci. China Technol. Sc. 53(1) (2010) 168-174.

[17] J. Schadlbauer, D. R. Walter, M. L. Husty, The 3-RPS parallel manipulator from an algebraic viewpoint, Mech. Mach. Theory 75 (2014) 161-176.

[18] Q.C. Li, Z. Chen, Q.H. Chen, C.Y. Wu, X.D. Hu, Parasitic motion comparison of 3-PRS parallel mechanism with different limb arrangements, 
Robot. Com.-Int Manuf. 27 (2011) 389-396.

[19] Y. M. Song, B. B. Lian, T. Sun, G. Dong, Y. Qi, H. Gao, A novel 5-DoF parallel manipulator and its kinematic optimization, J. Mech. Robot. 6 (4) 041008-1-041008-9.

[20] B. S. El-Khasawneh, P. M. Ferreira, Computation of stiffness bounds for parallel link manipulators, Int. J. Mach. Tools Manuf. 39 (1999) 321-342.

[21] D. Zhang, F. F. Xia, C. M. Mechefskec, S. Lang, Analysis of parallel kinematic machine with kinetostatic modelling method, Robot. Com.-Int Manuf. 20 (2004) 151-165.

[22] Y. Shneor, V.T. Portman, Stiffness of 5-axis machines with serial, parallel, and hybrid kinematics: Evaluation and comparison, CIRP Ann. Manuf. Techn. 59 (2010) 409-412.

[23] A. Pashkevich, D. Chablat, P. Wenger, Stiffness analysis of overconstrained parallel manipulators, Mech. Mach. Theory 44 (2009) 966-982.

[24] A. Klimchik, D. Chablat, A. Pashkevich, Stiffness modeling for perfect and non-perfect parallel manipulators under internal and external loadings, Mech. Mach. Theory 79 (2014) 1-28.

[25] D. Deblaise, X. Hernot, P. Maurine, A systematic analytical method for PKM stiffness matrix calculation, Proc. of the 2006 IEEE International Conference on Robotics and Automation, Florida, USA, 2006, pp. 4213-4219.

[26] J. Wu, J. S Wang, L.P. Wang, T. M. Li, Z. You, Study on the stiffness of a 5-DOF hybrid machine tool with actuation redundancy, Mech. Mach. Theory 44 (2009) 289-305.

[27] A. Klimchik, A. Pashkevich, D. Chablat, CAD-based approach for identification of elasto-static parameters of robotic manipulators, Finite Elem. Anal. Des. 75 (2013) 19-30.

[28] J. K. Salisbury, Active stiffness control of a manipulator in Caresian Coordinates, IEEE Conference on Decision and Control including the Symposium on Adaptive Processes, 1980, pp. 95-100.

[29] C. M. Gosselin, Stiffness mapping for parallel manipulators, IEEE T Robotic. Autom. 6(3) (1990) 377-382.

[30] C. Huang, W. H. Hung, I. Kao, New conservative stiffness mapping for the Stewart-Gough platform, Proceedings of the 2002 IEEE International Conference on Robotics and Automation, Washington, DC, USA, 2002, pp. 823-828.

[31] M. Ceccarelli, G. Carbone, A stiffness analysis for CaPaMan (CassinoParallel Manipulator), Mech. Mach. Theory 37 (2002) $427-439$.

[32] Y. M. Li, Q. S. Xu, Stiffness analysis for a 3-PUU parallel kinematic machine, Mech. Mach. Theory 43 (2008) 186-200.

[33] Y. M. Li, Q. S. Xu, An investigation on mobility and stiffness of a 3-DOF translational parallel manipulator via screw theory, Robot. Com.-Int Manuf. 24 (3) (2008) 402-414.

[34] Y.Y. Wang, H. T. Liu, T. Huang, D. G. Chetwynd, Stiffness modeling of the Tricept robot using the overall Jacobian matrix, J. Mech. Robot. 1 (2009) 021002-1-021002-8.

[35] J.S. Dai, X.L .Ding, Compliance analysis of a three-legged rigidly-connected platform device, J. Mech. Des. 128 (2006) $755-764$.

[36] F. Majou, C. M. Gosselin, P. Wenger, D. Chablat, Parametric stiffness analysis of the Orthoglide, Mech. Mach. Theory 42 (2007) $296-311$.

[37] V.T. Portman, Stiffness Evaluation of machines and robots: minimum collinear stiffness value approach, J. Mech. Robot. 3 (2011) 011015-1-011015-9.

[38] A. Pashkevich, A. Klimchik, D. Chablat, Enhanced stiffness modeling of manipulators with passive joints, Mech. Mach. Theory 46 (2011) 662-679.

[39] B. J. Yi, R. A. Freeman, Geometric analysis antagonistic stiffness redundantly actuated parallel mechanism, J. Robot. Sys. 10 (5) (1993) 581-603.

[40] T. S. Zhao, Y. Z. Zhao, H. Bian, N. Li, Continuous stiffness nonlinear mapping of spatial parallel mechanism, Chin. J. Mech. Eng. 44(8) (2008) 20-25. 